\title{
Double-charming Higgs boson identification using machine-learning assisted jet shapes
}

\author{
Alexander Lenz, ${ }^{1, *}$ Michael Spannowsky, ${ }^{1, \dagger}$ and Gilberto Tetlalmatzi-Xolocotzi ${ }^{2, \ddagger}$ \\ ${ }^{1}$ IPPP, Department of Physics, Durham University, Durham DH1 3LE, United Kingdom \\ ${ }^{2}$ Nikhef, Science Park 105, Amsterdam NL-1098 XG, Netherlands
}

(Received 12 October 2017; published 9 January 2018)

\begin{abstract}
We study the possibility of identifying a boosted resonance that decays into a charm pair against different sources of background using QCD event shapes, which are promoted to jet shapes. Using a set of jet shapes as input to a boosted decision tree, we find that observables utilizing the simultaneous presence of two charm quarks can access complementary information compared to approaches relying on two independent charm tags. Focusing on Higgs associated production with subsequent $H \rightarrow c \bar{c}$ decay and on a $C P$-odd scalar $A$ with $m_{A} \leq 10 \mathrm{GeV}$ we obtain the limits $\operatorname{Br}(H \rightarrow c \bar{c}) \leq 6.48 \%$ and $\mathcal{B r}(H \rightarrow A(\rightarrow c \bar{c}) Z) \leq 0.01 \%$ at $95 \%$ C.L.
\end{abstract}

DOI: 10.1103/PhysRevD.97.016001

\section{INTRODUCTION}

After the discovery of the Higgs boson [1,2] a precise determination of its couplings is now of fundamental importance. The couplings of the Higgs boson to the $W$ and $Z$ bosons are already known to be in good agreement with the standard model (SM) expectation, as can be inferred from the measurements of the Higgs decay and production rates by ATLAS and CMS [3-5].

In the SM the Yukawa interaction describes the coupling of the Higgs boson to a fermion $f$ with a strength given by the Yukawa coupling $y_{f}^{\mathrm{SM}}$. Deviations from the SM expectation can be parametrized by $\kappa_{f}=y_{f} / y_{f}^{\mathrm{SM}}$, which can be deduced from a measurement of the signal (S) strength $\mu_{f}$ defined as $\mu_{f}=\sigma_{H} \mathrm{Br}_{f \bar{f}} /$ $\left(\sigma_{H}^{\mathrm{SM}} \mathrm{Br}_{f \bar{f}}^{\mathrm{SM}}\right)$. Here $\sigma_{H}$ is the Higgs boson production cross section and $\mathrm{Br}_{f \bar{f}}$ is the branching ratio of the decay process $H \rightarrow f f$. Currently the couplings between the Higgs boson and the third generation fermions are consistent with the SM expectations; one gets $\mu_{t}=2.2 \pm$ 0.6 [6] (see [7] for slightly older values), $\mu_{b}=0.97 \pm$ 0.20 (weighted average based on the results presented in $[8,9])$ and $\mu_{\tau}=0.98 \pm 0.18$ [10]. However, much less is known about the couplings of the Higgs boson to fermions of the first two families: the current bounds

\footnotetext{
*alexander.lenz@durham.ac.uk michael.spannowsky@durham.ac.uk gtx@ nikhef.nl
}

Published by the American Physical Society under the terms of the Creative Commons Attribution 4.0 International license. Further distribution of this work must maintain attribution to the author(s) and the published article's title, journal citation, and DOI. Funded by SCOAP ${ }^{3}$. found by ATLAS [11] and CMS [12] are $\mu_{e} \leq 4 \times 10^{5}$ and $\mu_{\mu} \leq 7$. During the LHC's high-luminosity run $\mu_{\mu} \simeq 1$ might be achievable [13], while the electron coupling to the Higgs boson is far below the experimental sensitivity. Here a future $e^{+} e^{-}$collider could get close to the SM value $[14,15]$.

In this paper we focus on the coupling of the charm quark to the Higgs boson. Besides a measurement of the exclusive branching ratio $H \rightarrow J / \psi \gamma$ [16], yielding $\kappa_{c} \leq$ 220 [17-20], inclusive $H \rightarrow c \bar{c}$ decays were studied, e.g., in $[7,19,21-23]$.

A global fit to Higgs signal strengths gives the bound of $\kappa_{c} \leq 6.2$ [7]. In [24] a method based on Higgs transverse momentum distributions and using LHC Run I data leads to the following interval: $\kappa_{c} \in[-16,18]$. Modifications of the charm Yukawa coupling can occur in different new physics models [25-30]; it can even be 0 [6]. Our aim is to develop a strategy that allows us to set a direct upper limit on the charm Yukawa coupling.

The improvement in our bounds on $\mu_{c}$, derived from inclusive analyses, depends strongly on the c-tagging efficiency at the LHC. While dedicated charm tagging algorithms are relatively new [31], flavor tagging has been used in the identification of jets derived from the hadronization of $b$ quarks for more than 20 years, and was employed at the Tevatron for the discovery of the top quark $[32,33]$. Two features of the b-mesons are exploited to achieve a good b-tagging performance: (1) the dominance of semileptonic rates when a b-hadron decays and (2) the long lifetime of $b$-hadrons. For the latter one can search for displaced secondary vertices (decay vertex) of $b$-hadrons with respect to the primary vertex (interaction point) in a given event. This distance, known as impact parameter, is normally larger for $b$-hadrons in comparison 
with that associated with states obtained from the hadronization of light quarks $(u, d, s)$ and gluons. A similar approach can be followed for $c$-jets. However, as tagging procedures for $b$-jets and $c$-jets are quite similar, their mutual misidentification rates are consequently quite large.

In general, bottom or charm taggers are designed to find jets initiated by individual $b$ or $c$ quarks, allowing for a generic use of these algorithms in a wide range of applications. However, in searches for light or boosted resonances that decay into a charm or bottom pair, such algorithms might not be ideal, as they neglect correlations between the decay products. For example, if the decaying resonance is a color singlet particle, its decay products are color connected and soft gluon emissions of either decay product have a preference to be emitted into the cone between the quark pair [34]. Thus to increase the sensitivity in searches for new physics or Higgs boson measurements it can be beneficial to design dedicated two-prong reconstruction algorithms that allow one to utilize more information about the decaying resonances. Observables that are particularly sensitive to the radiation profile of the event are so-called event shape observables [35,36], which have been proposed as a hypothesis tester in the study of Higgs boson properties [37,38]. By promoting those well-studied observables to jet shape observables, applied to a fat jet, they can be used as input to machine-learning algorithms to separate signal from large QCD backgrounds (B).

In this paper we present a procedure to identify jets initiated by $c \bar{c}$ pairs from Higgs boson decays based on the application of different event shapes and the transverse momenta of leptons $\left(e^{ \pm}\right.$and $\left.\mu^{ \pm}\right)$. It is expected that high- $p_{T}$ jets arising from highly boosted Higgs bosons have a different energy flow in comparison to jets arising from pure QCD backgrounds. We emphasize that in the double tagging strategy presented in this work, we study the energy distribution of the full jet associated with the boosted Higgs bosons decaying into the $\mathrm{c}$ and the $\bar{c}$ quark, without separating the corresponding subjets after hadronization. Our analysis is based on fully showered and hadronized Monte Carlo events and the results obtained can be considered as an upper bound to a more complete study when detector effects are also included.

The structure of the paper is as follows: In Sec. II we describe the event generation and the selection criteria. Then in Sec. III we present the performance of our approach for the selection of the SM Higgs boson $H$ against different sources of background. Using the tagging efficiencies derived from the optimization against QCD c-jets and jets originated from the transition $H \rightarrow b \bar{b}$, we present an upper bound for our sensitivity to $\mathcal{B} r(H \rightarrow c \bar{c})$. In order to evaluate the efficiency of the simultaneous double c-tagging identification with strategies based on the double application of a single c-tagger, we compare our results with those obtained applying the Atlas single charm tagging algorithm JetFitterCharm. Section IV is devoted to the study of the decay channel $H(\rightarrow A(\rightarrow c \bar{c}) Z)+$ jets, with $A$ being the two Higgs doublet model (THDM) $C P$ odd scalar. Finally in Sec. V we conclude. The discussion is complemented with the correlation matrices among the event shapes used as well as with the distributions for the leading ones in each one of our studies. A brief description of most of the observables considered is included in the appendix.

The analytical determination of correlations between jet shapes labeled as "generalized angularities" has been exemplified up to next-to-leading-logarithmic (NLL) order in [39]. It uses the technology of double differential cross sections with respect to pairs of discrimination observables $[40,41]$. One of the advantages of using this procedure is not only the possibility of assessing how correlated two variables are, but also the fact that it allows us to evaluate the "truth" overlap between them. This is the amount of relevant information useful for the quark/gluon discrimination when both of the observables are included. In our analysis we follow a more pedestrian approach by

TABLE I. Top observables determined by the multivariate analysis to discriminate the process $p p \rightarrow H(\rightarrow c \bar{c}) Z$ against the combination $p p \rightarrow Z+$ jets and $p p \rightarrow H(\rightarrow b \bar{b}) Z$.

Signal: $H(\rightarrow c \bar{c}) Z$

Backgrounds: $Z+$ jets and $H(\rightarrow b \bar{b}) Z$

\begin{tabular}{lll}
\hline Nonleptonic & \multicolumn{1}{c}{ Single leptonic } & \multicolumn{1}{c}{ Double leptonic } \\
\hline $\begin{array}{l}\text { Cone heavy Jet mass } \\
\text { C parameter }\end{array}$ & $\begin{array}{l}\text { Cone total Jet mass } \\
\text { Thrust major }\end{array}$ & C parameter \\
& Thrust major & Thrust major \\
$P_{T, e}$ & $\begin{array}{c}\text { Global thrust minor defined with jets } \\
\text { outside the dijet region }\end{array}$ \\
3-jet resolution $y_{3}$ Geneva (E0-scheme) & 3-jet resolution $y_{3}$ Durham (P-scheme) & C parameter \\
3-jet resolution $y_{3}$ Durham (P-scheme) & $\begin{array}{l}\text { Global thrust minor defined with } \\
\text { jets outside the dijet region }\end{array}$ & 3-jet resolution $y_{3}$ Durham (P-scheme) \\
$\begin{array}{l}\text { Global thrust minor defined with jets } \\
\text { outside the dijet region }\end{array}$ & $P_{T, \mu}$ & $P_{T, \mu}$ \\
Transverse sphericity & $P_{T, e}$ & Transverse sphericity \\
\hline \hline
\end{tabular}




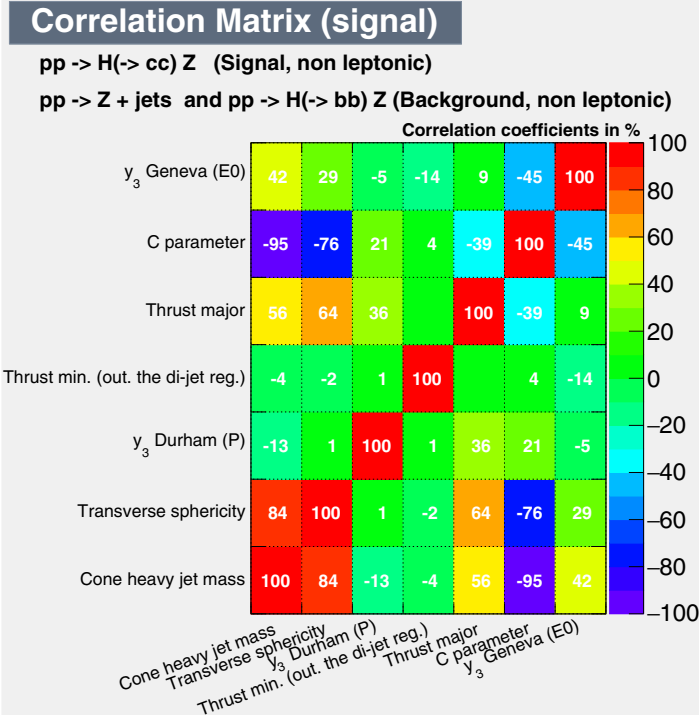

\section{Correlation Matrix (signal)}

\section{pp $\rightarrow \mathrm{H}(->\mathrm{cc}) \mathrm{Z}$ (Signal, single leptonic)}

$\mathrm{pp}->\mathrm{Z}+$ jets and $\mathrm{pp}->\mathrm{H}(->\mathrm{bb}) \mathrm{Z}$ (Background, single leptonic)

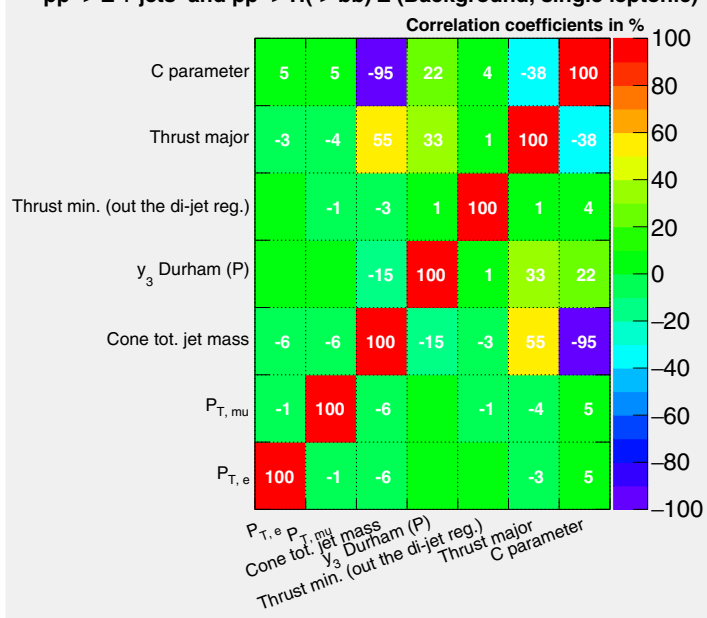

\section{Correlation Matrix (signal)}

pp $->\mathrm{H}(->\mathrm{cc}) \mathrm{Z}$ (Signal, double leptonic)

$\mathrm{pp}->\mathrm{Z}+$ jets and $\mathrm{pp}->\mathrm{H}(->\mathrm{bb}) \mathrm{Z}$ (Background,double leptonic)

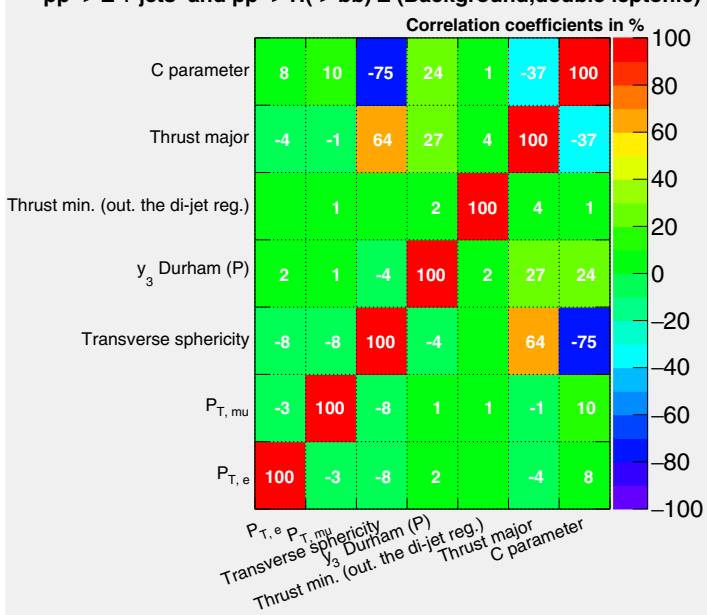

\section{Correlation Matrix (background)}

pp $->\mathrm{H}(->\mathrm{cc}) \mathrm{Z}$ (Signal, non leptonic)

pp $->Z+$ jets and pp $->H(->b b) Z$ (Background, non leptonic)

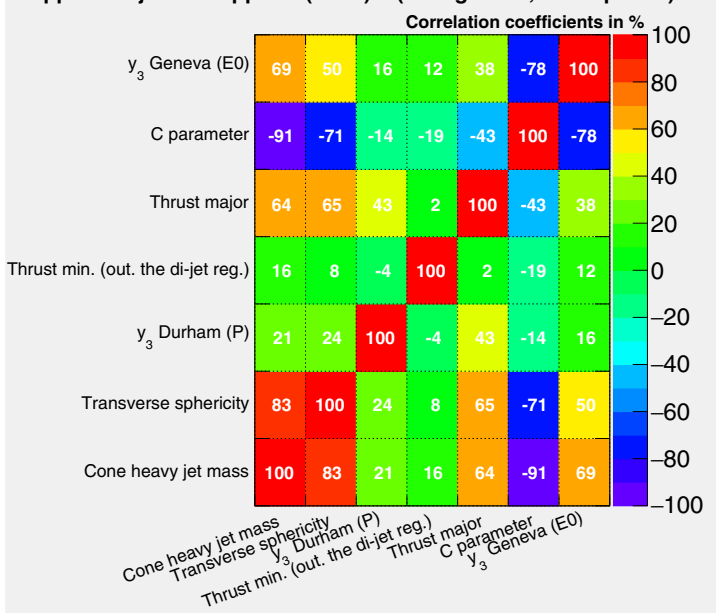

\section{Correlation Matrix (background)}

$\mathrm{pp}->\mathrm{H}(->\mathrm{cc}) \mathrm{Z}$ (Signal, single leptonic)

pp $->Z+$ jets and pp $->H(->b b) Z$ (Background, single leptonic)

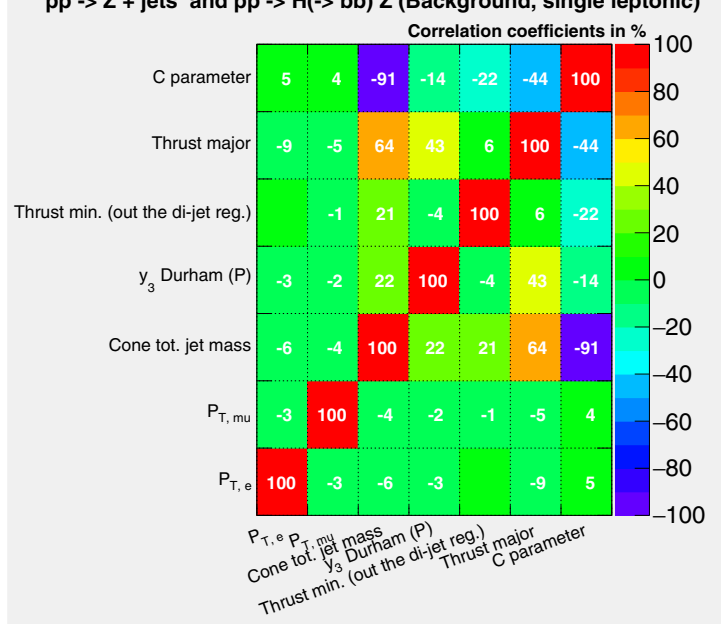

\section{Correlation Matrix (background)}

pp $->\mathrm{H}(->\mathrm{cc}) \mathrm{Z}$ (Signal, double leptonic)

pp $->Z+$ jets and pp $->\mathrm{H}(->$ bb) $Z$ (Background,double leptonic)

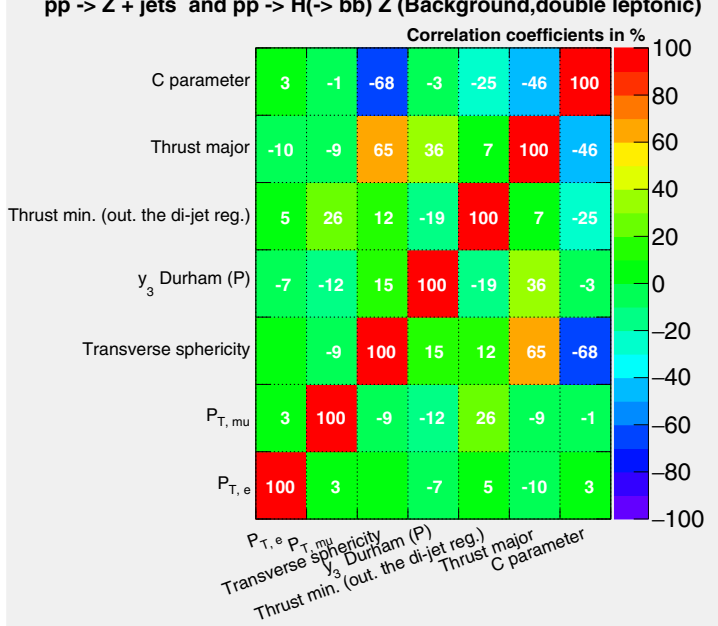

FIG. 1. Correlation coefficients for the observables used in the analysis $p p \rightarrow H(\rightarrow c \bar{c}) Z$ (signal) vs the combination $p p \rightarrow Z+$ jets and $p p \rightarrow H(\rightarrow b \bar{b}) Z$ (background). 

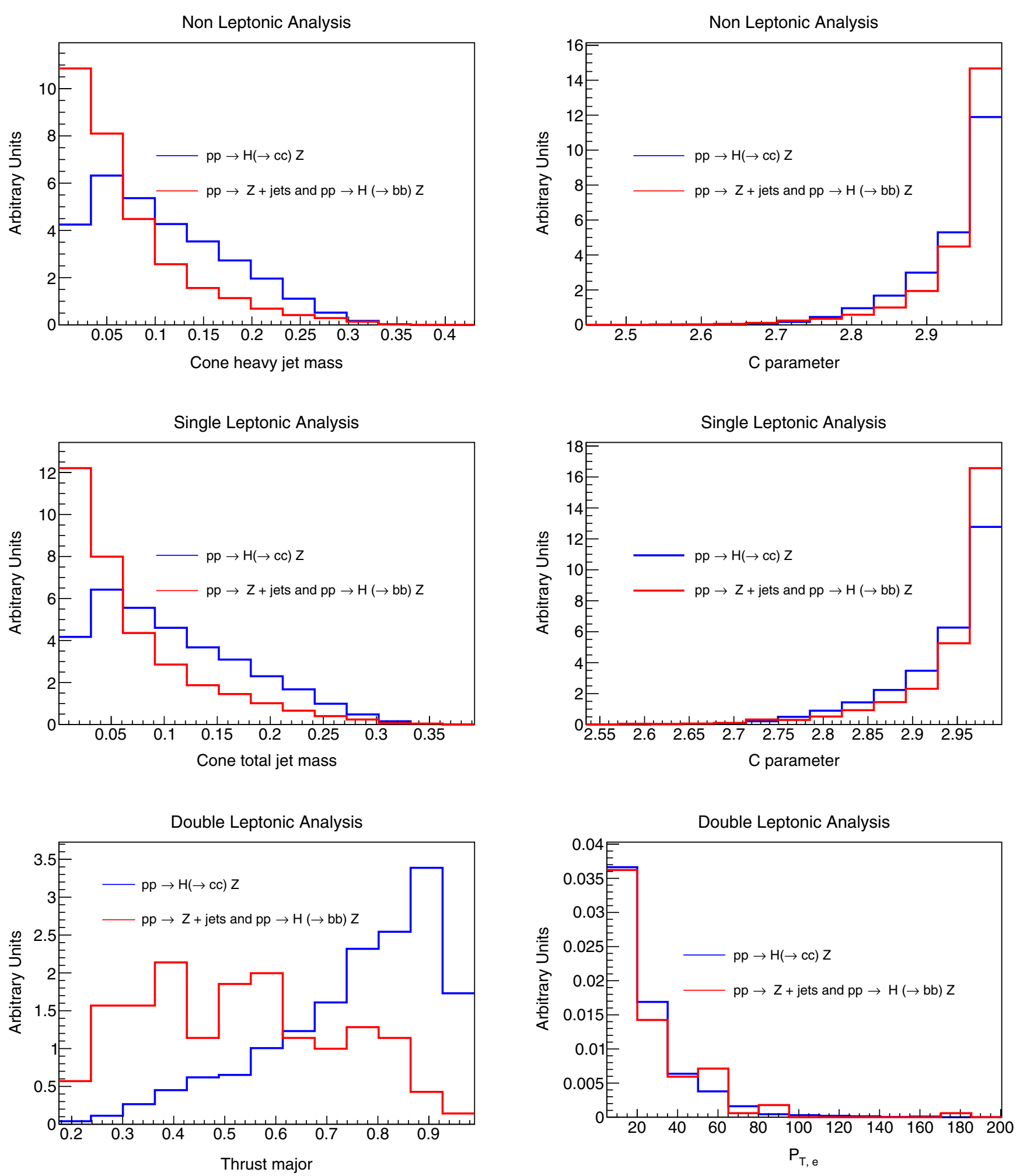

FIG. 2. Histograms for the main discrimination event shapes when selecting $p p \rightarrow H(\rightarrow c \bar{c}) Z$ against the combination $p p \rightarrow$ $Z+$ jets and $p p \rightarrow H(\rightarrow b \bar{b}) Z$. 


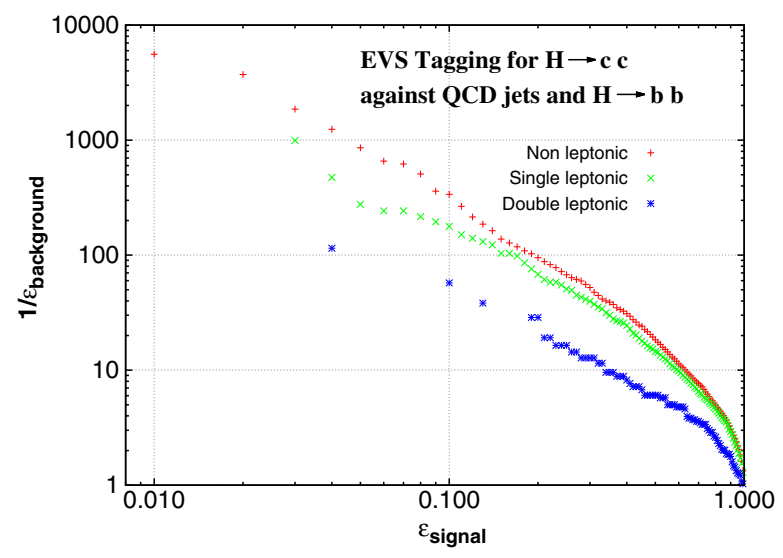

FIG. 3. Double $c$-jet selection efficiency (from $H \rightarrow c \bar{c}$ ) against the QCD-jet rejection achieved by the event-shapes tagger. The curves are obtained from independent optimizations considering different subsamples with zero (nonleptonic), one (single leptonic), and two (double leptonic) leptons inside the highest transverse momentum fat jet.

combining the information provided by the correlation matrices obtained from a multivariate analysis and the discrimination power of the observables evaluated using the receiver operating characteristic (ROC) curves. A study along the lines of the references quoted is out of the scope of the present paper. Additionally the decorrelation between the jet mass and jet-substructure observables has been discussed in [42-44].

\section{EVENT GENERATION AND EVENT SELECTION}

The signal channels are $p p \rightarrow H(\rightarrow c \bar{c}) Z$ and $p p \rightarrow H(\rightarrow A(\rightarrow c \bar{c}) Z)+$ jets. Here $H$ is the SM Higgs boson and $A$ denotes the $C P$-odd THDM scalar. As the background channel we include $p p \rightarrow Z+$ jets and $p p \rightarrow H(\rightarrow b \bar{b}) Z$. In all cases we consider $Z \rightarrow l^{+} l^{-}$, for $l=e, \mu$. We take into account two possible values for the mass of the scalar $A, m_{A}=4 \mathrm{GeV}$ and $m_{A}=10 \mathrm{GeV}$. We generate our samples with SHERPA 2.2.1 [45] at $\sqrt{s}=13.0 \mathrm{TeV}$, and include parton shower, hadronization, and underlying event contributions. For the jet reconstruction we use the jet finding package FastJet 3.2.1 [46]. The event selection is performed with the version 2.4.2 of the RIVET analysis framework [47].

TABLE II. Fraction of events in each one of the leptonic categories for the samples $p p \rightarrow H(\rightarrow c \bar{c}) Z$ and the admixture of the channels $p p \rightarrow Z+$ jets and $p p \rightarrow H(\rightarrow b b) Z$ after the selection cuts.

\begin{tabular}{lcc}
\hline \hline Fraction of events & $p p \rightarrow H(\rightarrow c \bar{c}) Z$ & $\begin{array}{c}p p \rightarrow Z+\text { jets and } \\
p p \rightarrow H(\rightarrow b \bar{b}) Z\end{array}$ \\
\hline$f^{(0)}$ (0 leptons) & $73.62 \%$ & $78.42 \%$ \\
$f^{(1)}$ (1 lepton) & $24.47 \%$ & $19.55 \%$ \\
$f^{(2)}$ (2 leptons) & $1.90 \%$ & $2.03 \%$ \\
\hline \hline
\end{tabular}

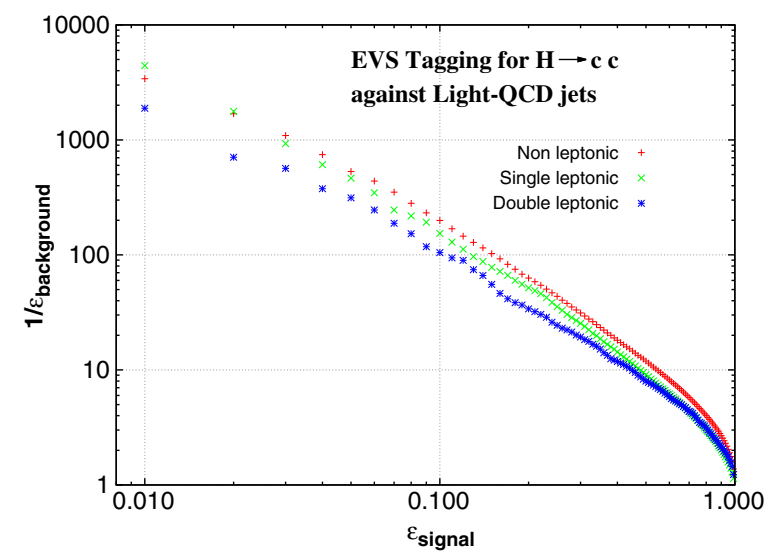

FIG. 4. Double $c$-jets selection efficiency against the double light jets rejection achieved by the event-shapes tagger per leptonic study.

Our selection strategy is based on the identification of the Higgs and a $Z$ boson in the highly boosted regime, when both particles have a large transverse momentum and are back to back. In order to reconstruct the $Z$ boson we require two isolated leptons $l^{+} l^{-}$(for $l=e, \mu$ ) with a combined mass satisfying $80.0 \mathrm{GeV}<m_{l l}<100.0 \mathrm{GeV}$. A lepton $l$ is considered isolated if the following inequality is satisfied: $E_{l} / E_{R}<0.1$, where $E_{l}$ is the energy of $l$ and $E_{R}$ is the total energy inside a cone of radius $R=0.3$ around $l$. The identification of the $Z$ boson concludes by imposing a cut $p_{T}>200.0 \mathrm{GeV}$ over the combined transverse momentum of the pair $l^{+} l^{-}$. We proceed with the next steps only if the $\mathrm{Z}$ boson has been successfully reconstructed as described in this paragraph.

A boosted Higgs boson decaying into a pair of quarks $q \bar{q}$ produces a jet with a relatively large active area $R_{q \bar{q}}$, and thus is commonly referred to as a fat jet. As a matter of fact, in the boosted regime, the radius of the jet depends on the mass and the transverse momentum of the Higgs boson

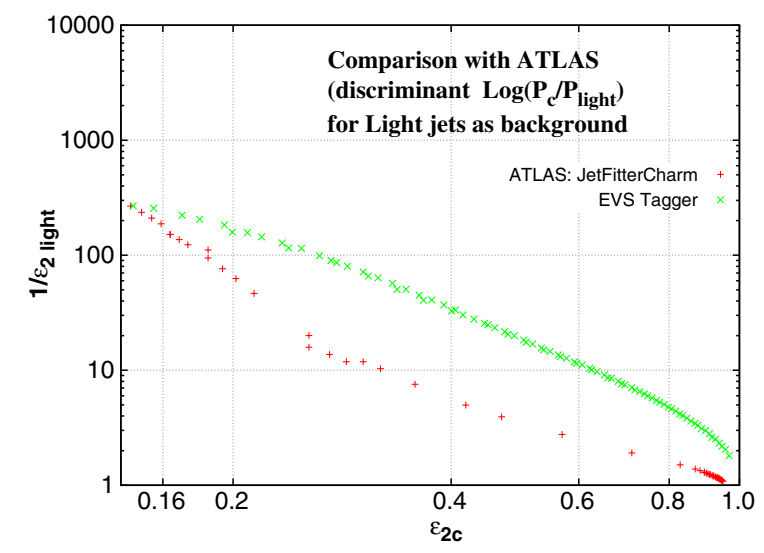

FIG. 5. Double $c$-jets selection efficiency against the double light jets rejection achieved by the ATLAS JetFitterCharm tagger and the combined event-shapes tagger. 
TABLE III. Top observables determined by the multivariate analysis to discriminate the process $p p \rightarrow H(\rightarrow c \bar{c}) Z$ against $p p \rightarrow Z+$ light jets.

Signal: $H(\rightarrow c \bar{c}) Z$

Background: $Z+$ light quark jets

\begin{tabular}{lll}
\hline Nonleptonic & \multicolumn{1}{c}{ Single leptonic } & \multicolumn{1}{c}{ Double leptonic } \\
\hline Fox Wolfram-like $n=1 / 4$ & Fractional energy correlation $x=1.5$ & Fractional energy correlation $x=1.5$ \\
3-jet resolution $y_{3}$ Durham (P-scheme) & C parameter & $P_{T, e}$ \\
C parameter & 3-jet resolution $y_{3}$ Jade (E-scheme) & 3-jet resolution $y_{3}$ Jade (E-scheme) \\
Thrust of $e^{-|\eta|}$ momenta & 4-jet resolution $y_{4}$ Durham (P-scheme) & 3-jet resolution $y_{3}$ Geneva (P-scheme) \\
3-jet resolution $y_{3}$ Jade (E-scheme) & $P_{T, \mu}$ & C parameter \\
3-jet resolution $y_{3}$ Geneva (P-scheme) & $P_{T, e}$ & 3-jet resolution $y_{3}$ Durham (P-scheme) \\
3-jet resolution $y_{3}$ Jade (E0-scheme) & 3-jet resolution $y_{3}$ Geneva (P-scheme) & $P_{T, \mu}$ \\
\hline \hline
\end{tabular}

$\left(m_{H}\right.$ and $\left.p_{T, H}\right)$ as well as on the momentum fractions of the quark and the antiquark ( $\mathrm{z}$ and $1-z$ ) according to $R_{q \bar{q}}=m_{H} /\left(p_{T, H} \sqrt{z(1-z)}\right)$. Thus, for a Higgs boson of mass $m_{H} \simeq 125 \mathrm{GeV}$ and a transverse momentum $p_{T} \simeq$ $200 \mathrm{GeV}$ decaying symmetrically into a pair charm anticharm, we expect an angular separation of the Higgs decay products of $R_{c \bar{c}} \simeq 1.25$. In practice we demand jets with radius $R=1.2$ and a transverse momentum $p_{T}>200 \mathrm{GeV}$ reconstructed, with the anti- $k_{T}$ algorithm and select the jet with the highest $p_{T}$. We translate all the constituents of this jet to the plane $\eta=0$ by taking $p_{z}=0$ and replacing their total energy by their corresponding transverse energy [48].

From next-to-leading order-QCD calculations, in the SM the decay fractions of c-quarks into leptons obey with good approximation [49] $\mathcal{B r}\left(c \rightarrow \bar{l}_{\nu_{l}} X\right)=(21.74 \pm 3.90) \%$ and $\mathcal{B} r\left(c \rightarrow X^{\prime}\right)=100 \%-\mathcal{B} r\left(c \rightarrow \bar{l} \nu_{l} X\right)$ where $\bar{l}=\bar{e}, \bar{\mu}$ and $X, X^{\prime}$ denote quark final states. Hence, if we consider jets originated from the hadronization process of $c \bar{c}$ pairs, we can expect to find 0,1 , and 2 leptons with the following probabilities: $61.24 \%, 34.03 \%$, and $4.73 \%$, respectively. For each one of our analyses we perform three independent studies: nonleptonic, single leptonic, and double leptonic, if zero, one, and two nonisolated leptons are found inside the fat jet, respectively. A cut in the transverse momentum of the leptons of $p_{T, l} \geq 2.0 \mathrm{GeV}$ allows us to reproduce these numbers with good approximation. Nevertheless, we consider this to be a relatively soft cut; hence in practice we impose the constraint $p_{T, l} \geq 5.0 \mathrm{GeV}$.

If an event is selected, we probe the substructure of the highest $p_{T}$ fat jet by applying a collection of different event shapes on its constituents, thereby promoting the event shapes to jet shapes. We follow this procedure separately for each one of the leptonic categories introduced in the previous paragraph. To evaluate the signal efficiency and mistag rate of our observables we use a multivariate analysis implemented in the toolkit for multivariate analysis (TMVA) package [50] and consider a boosted decision tree (BDT) as our classifier. In addition to the event shapes and for the single-leptonic and double-leptonic categories, we also include the value of the transverse momentum of the highest $p_{T}$ light lepton found inside the selected fat jet.

\section{STANDARD MODEL HIGGS $c \bar{c}$ TAGGING USING EVENT SHAPES}

\section{A. Performance}

We begin by obtaining the performance of our strategy when selecting the signal channel $p p \rightarrow H(\rightarrow c \bar{c}) Z$ against the combined admixture of backgrounds $p p \rightarrow$ $Z+$ jets and $p p \rightarrow H(\rightarrow b \bar{b}) Z$. The set of observables that gives us the best performance is presented in Table I and the correlations among them are shown in Fig. 1; additionally we provide the distributions for the top two discriminating observables in each one of the leptonic categories in Fig. 2.

Our curves for the signal selection efficiencies as well as our background fake rates in each one of the leptonic studies are shown in Fig. 3. We can combine the three leptonic studies to obtain a single selection efficiency for $\mathrm{S}$ and for $\mathrm{B}$ according to the formula

$\varepsilon_{S . / B .}^{\mathrm{Tot}}=\varepsilon_{S . / B .}^{(0)} \times f_{S . / B .}^{(0)}+\varepsilon_{S . / B .}^{(1)} \times f_{S . / B .}^{(1)}+\varepsilon_{S . / B .}^{(2)} \times f_{S . / B .}^{(2)}$.

Our optimal point after the combination of the different leptonic categories corresponds to

$$
\varepsilon_{c \bar{c}}=0.39 \quad \varepsilon_{\mathrm{QCD}, \mathrm{jets}}=0.03,
$$

obtained from the following partial efficiencies,

$$
\begin{array}{lll}
\varepsilon_{S .}^{(0)}=0.39, & \varepsilon_{S .}^{(1)}=0.39, & \varepsilon_{S .}^{(2)}=0.19 \\
\varepsilon_{B .}^{(0)}=0.03, & \varepsilon_{B .}^{(1)}=0.04, & \varepsilon_{B .}^{(2)}=0.03,
\end{array}
$$

and the leptonic fractions shown in Table II. Our branching ratio for the process $H \rightarrow c \bar{c}$ is then $\operatorname{Br}(H \rightarrow c \bar{c})=6.48 \%$ leading to the cross section $\sigma_{p p \rightarrow H(\rightarrow c \bar{c}) Z}=0.08 \mathrm{fb}$. In the case of the backgrounds the corresponding cross sections are $\sigma_{p p \rightarrow Z+\text { jets }}=23.56 \mathrm{fb}$ and $\sigma_{p p \rightarrow H(\rightarrow b \bar{b}) Z}=0.72 \mathrm{fb}$. Based on these results and considering the integrated 


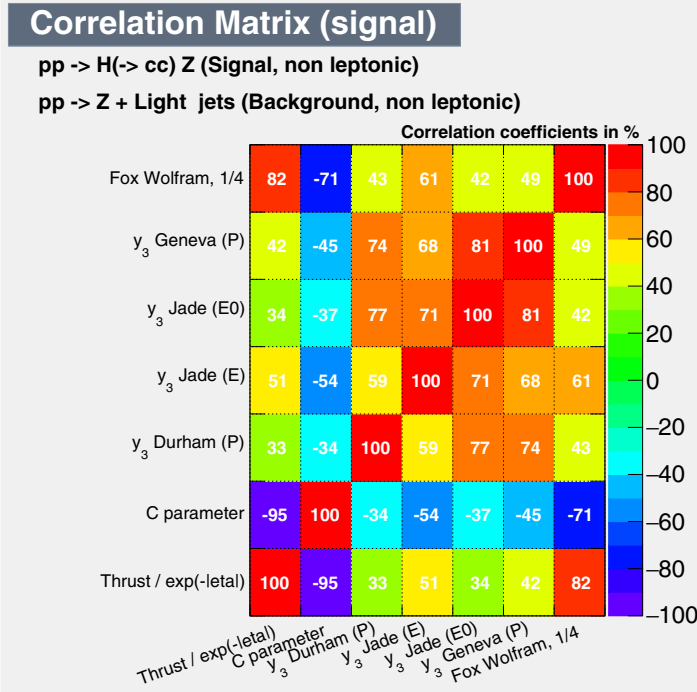

\section{Correlation Matrix (signal)}

pp $->\mathrm{H}(->\mathrm{cc}) \mathrm{Z}$ (Signal, single leptonic)

pp $->$ Z Light jets (Background, single leptonic)

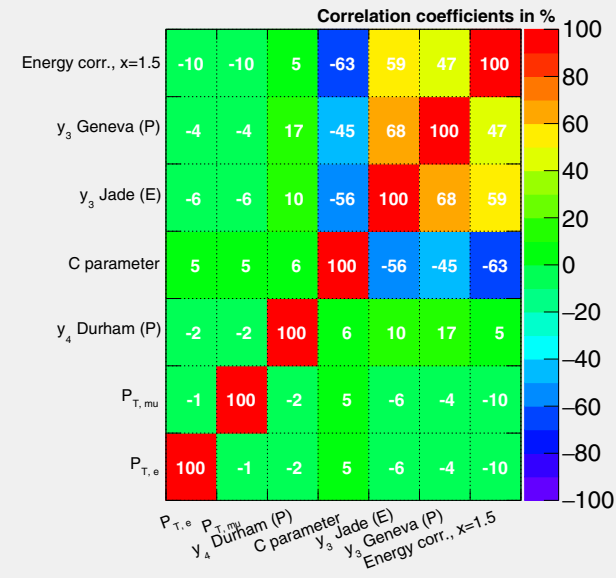

\section{Correlation Matrix (signal)}

pp $->\mathrm{H}(->\mathrm{cc}) \mathrm{Z}$ (Signal, double leptonic)

pp $->Z+$ Light jets (Background, double leptonic)

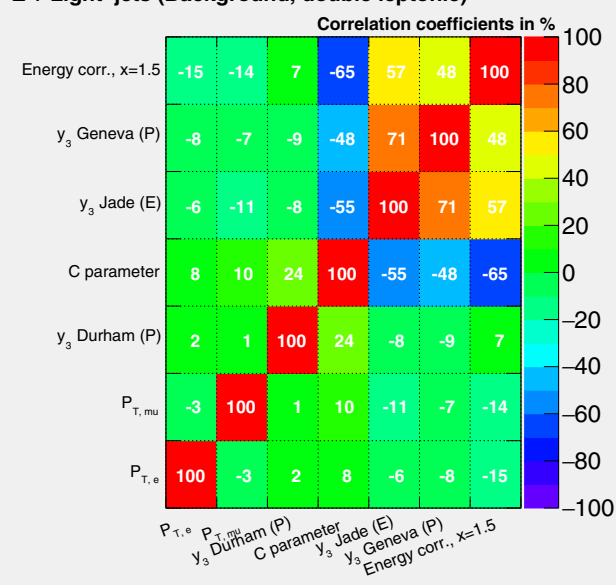

\section{Correlation Matrix (background)}

pp $->\mathrm{H}(->\mathrm{cc}) \mathrm{Z}$ (Signal, non leptonic)

$p p->Z+$ Light jets (Background, non leptonic)

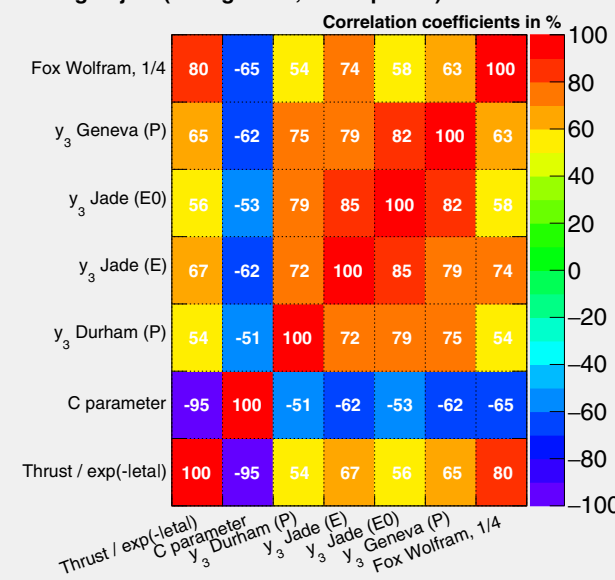

\section{Correlation Matrix (background)}

pp -> H(-> cc) Z (Signal, single leptonic)

pp $->Z+$ Light jets (Background, single leptonic)

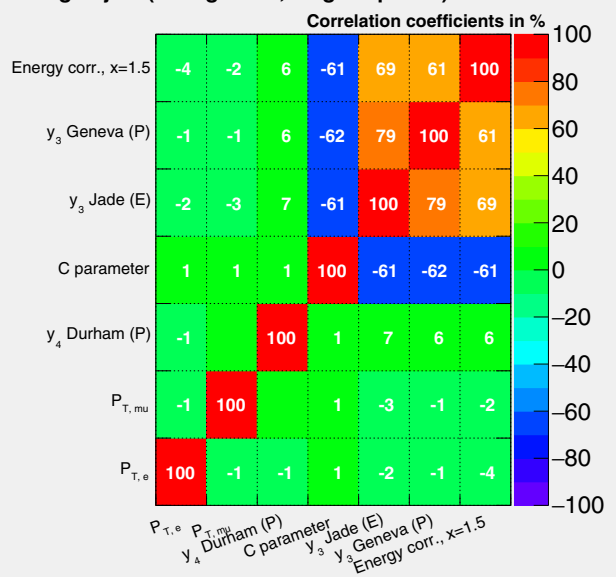

\section{Correlation Matrix (background)}

pp -> H(-> cc) Z (Signal, double leptonic)

pp -> Z + Light jets (Background, double leptonic)

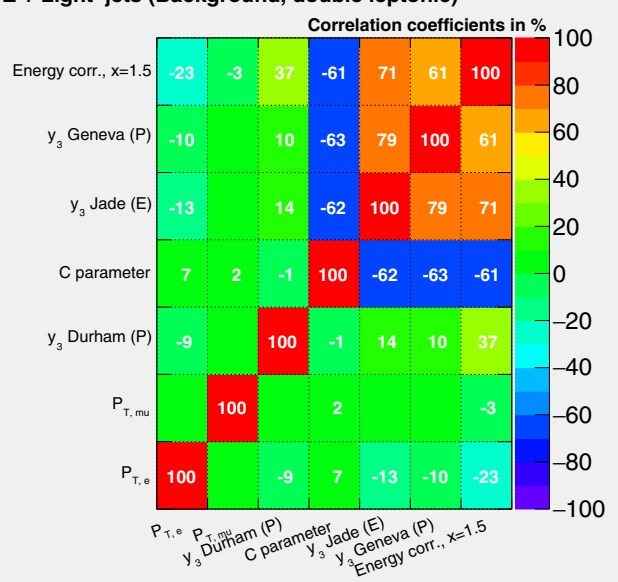

FIG. 6. Correlation coefficients for the observables used in the analysis $p p \rightarrow H(\rightarrow c \bar{c}) Z$ (signal) vs $p p \rightarrow Z+$ light jets (background). 


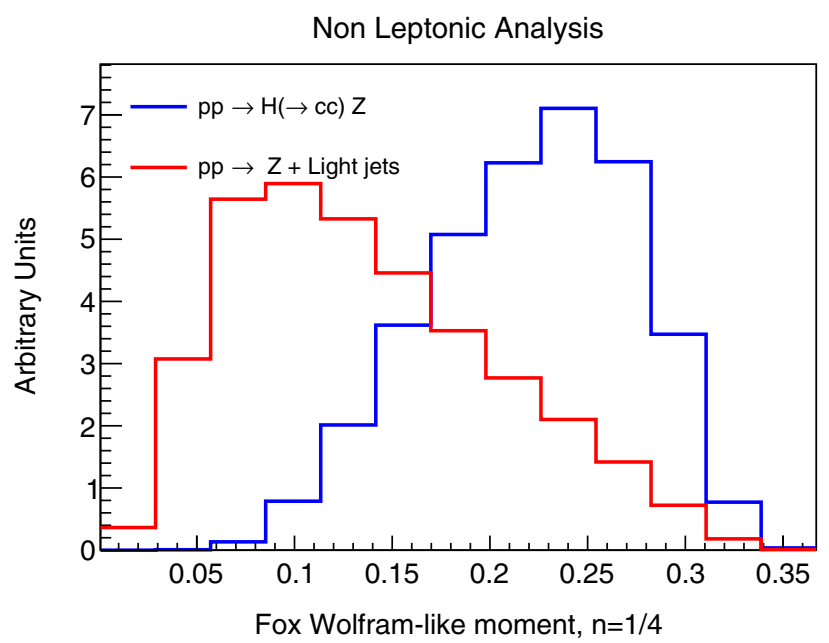

Non Leptonic Analysis
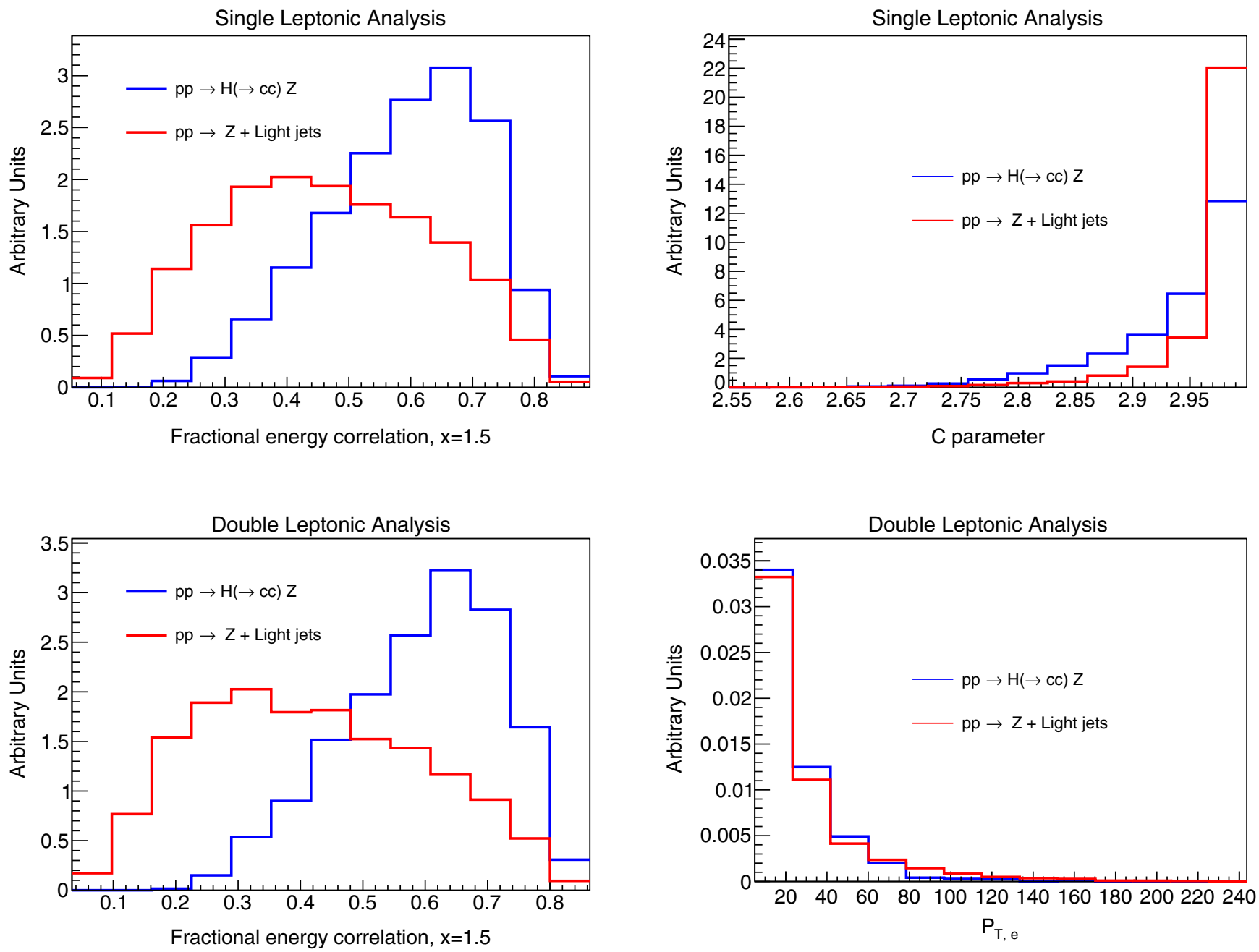

FIG. 7. Histograms for the main discrimination event shapes when selecting $p p \rightarrow H(\rightarrow c \bar{c}) Z$ against $p p \rightarrow Z+$ light jets. 
TABLE IV. Top observables determined by the multivariate analysis to discriminate the process $p p \rightarrow H(\rightarrow A(\rightarrow c \bar{c}) Z)+$ jets against $p p \rightarrow Z+$ jets for $m_{A}=4 \mathrm{GeV}$.

Signal: $C P$-odd THDM scalar A $(4 \mathrm{GeV})$

Background: $Z+$ jets

\begin{tabular}{lll}
\hline Nonleptonic & \multicolumn{1}{c}{ Single leptonic } & \multicolumn{1}{c}{ Double leptonic } \\
\hline Transverse spherocity & Transverse spherocity & Transverse spherocity \\
Fractional energy correlation $x=1.5$ & Fox Wolfram-like $n=1 / 4$ & Fractional energy correlation $x=1.5$ \\
Thrust major & Thrust major & 4-jet resolution $y_{4}$ Durham (P-scheme) \\
C parameter & 3-jet resolution $y_{3}$ Jade (E-scheme) & 3-jet resolution $y_{3}$ Jade (E-scheme) \\
Cone $y_{3}\left(k_{t}, \Delta R, E\right.$-scheme) & 3-jet resolution $y_{3}$ Durham (P-scheme) & $P_{T, \mu}$ \\
3-jet resolution $y_{3}$ Jade (P-scheme) & $P_{T, e}$ & $P_{T, e}$ \\
3-jet resolution $y_{3}$ Jade (E-scheme) & $P_{T, \mu}$ & 3-jet resolution $y_{3}$ Durham (E0-scheme) \\
\hline \hline
\end{tabular}

luminosity $\int \mathcal{L} d t=3000 \mathrm{fb}^{-1}$ we can verify the 2 sigma condition for the significance $\mathcal{S} / \sqrt{\mathcal{B}}=2.0$.

\section{B. Comparison against the ATLAS JetFitterCharm algorithm}

In order to evaluate the performance of our double charm identification approach against more conventional lifetime-based single charm tagging procedures, we provide a "naive" comparison with the ATLAS JetFitterCharm algorithm [31]. In the ATLAS study two main sources of backgrounds are considered, the first one being lightflavor jets, i.e., jets arising from the hadronization of $g, u, d, s, \bar{u}, \bar{d}, \bar{s}$; the second background is heavy-flavor jets, in this context $b$-jets.

From [31] we extract the JetFitterCharm single selection efficiencies $\epsilon_{c}, \epsilon_{b}$, and $\epsilon_{\text {light }}$ for the charm jets, b-jets, and light jets, respectively. The double tagging coefficients for each category are calculated as $\varepsilon_{2 c}=\epsilon_{c}^{2}, \varepsilon_{2 b}=\epsilon_{b}^{2}$, and $\varepsilon_{2 \text { light }}=\epsilon_{\text {light }}^{2}$.

For the comparison of the different tagging strategies, we used the boosted Higgs search described in Sec. II, where

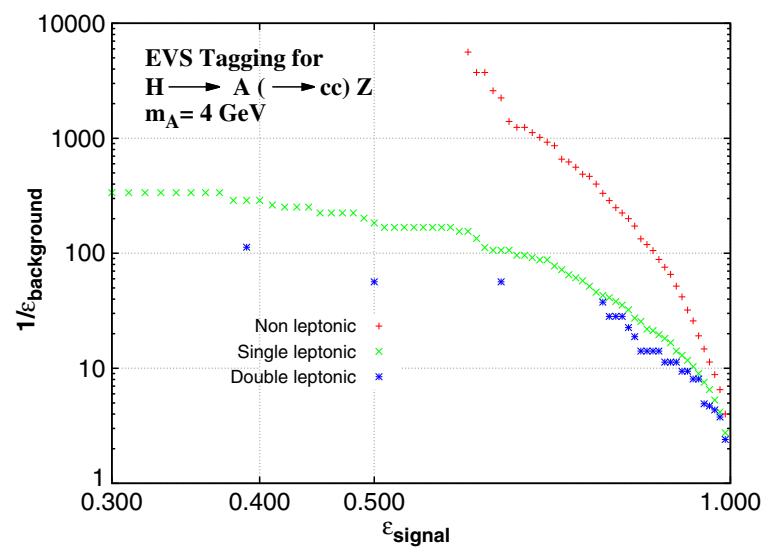

FIG. 8. Double $c$-jets selection efficiency (from $A \rightarrow c \bar{c}$ ) against the QCD-jets rejection achieved by the event-shapes tagger per leptonic study. Here we are considering $m_{A}=4 \mathrm{GeV}$.
TABLE V. Fraction of events in each one of the leptonic categories for the samples $p p \rightarrow H(\rightarrow A(\rightarrow c \bar{c}) Z)+$ jets and $p p \rightarrow Z+$ jets after the selection cuts for $m_{A}=10 \mathrm{GeV}$.

\begin{tabular}{lcc}
\hline \hline & $p p \rightarrow H+$ jets & \\
& with $H \rightarrow A(\rightarrow c \bar{c}) Z$ & \\
Fraction of events & $m_{A}=4 \mathrm{GeV}$ & $p p \rightarrow Z+$ jets \\
\hline$f^{(0)}$ (0 leptons) & $85.5 \%$ & $84.0 \%$ \\
$f^{(1)}$ (1 lepton) & $13.5 \%$ & $15.15 \%$ \\
$f^{(2)}$ (2 leptons) & $1.0 \%$ & $0.85 \%$ \\
\hline \hline
\end{tabular}

the dominant backgrounds are light-flavor jets $+Z$ and $b \bar{b}$ jets $+Z$. As the JetFitterCharm efficiencies are not provided in terms of separate analyses for the different leptonic categories introduced in Sec II, we combine the selection efficiencies achieved in our approach for the nonleptonic, single-leptonic, and double-leptonic studies for a given background according to Eq. (1).

We find the best results in rejecting light-flavor jets, which have in this analysis a cross section that is at least an order of magnitude bigger than the $b \bar{b}$ background.

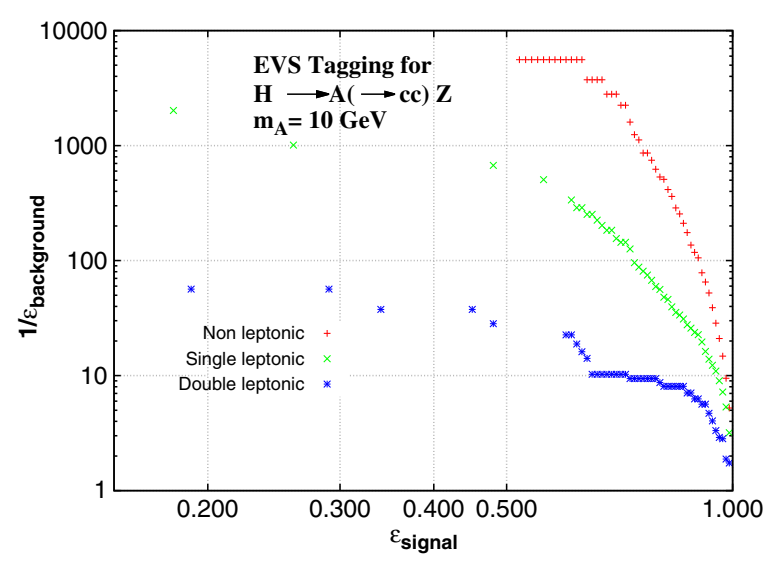

FIG. 9. Double $c$-jets selection efficiency (from $A \rightarrow c \bar{c}$ ) against the QCD-jets rejection achieved by the event-shapes tagger per leptonic study. Here we are considering $m_{A}=10 \mathrm{GeV}$. 
TABLE VI. Top observables determined by the multivariate analysis to discriminate the process $p p \rightarrow H(\rightarrow A(\rightarrow c \bar{c}) Z)+$ jets against $p p \rightarrow Z+$ jets for $m_{A}=10 \mathrm{GeV}$.

\section{$C P$-odd THDM scalar A $(10 \mathrm{GeV})$ \\ Background: $Z+$ jets}

\begin{tabular}{lll}
\hline Nonleptonic & \multicolumn{1}{c}{ Single leptonic } & \multicolumn{1}{c}{ Double leptonic } \\
\hline Transverse spherocity & Transverse spherocity & $P_{T, \mu}$ \\
Fox Wolfram-like $n=1 / 4$ & Fractional energy correlation $x=1.5$ & Thrust major \\
Directly global $y_{3}\left(k_{t}, \Delta R, E\right)$ & C parameter & $P_{T, e}$ \\
Thrust major & $P_{T, \mu}$ & Fractional energy correlation $x=1.5$ \\
C parameter & 3-jet resolution $y_{3}$ Jade (E-scheme) & 3-jet resolution $y_{3}$ Jade (P-scheme) \\
3-jet resolution $y_{3}$ Jade (E-scheme) & $P_{T, e}$ & C parameter \\
& 3-jet resolution $y_{3}$ Durham (P-scheme) & 4-jet resolution $y_{4}$ Durham (P-scheme) \\
\hline \hline
\end{tabular}

In Fig. 4 we show the ROC curves for the different leptonic analyses and in Fig. 5 we present the performance obtained from the combination of the leptonic categories. The observables giving to us the best performance are summarized on Table III, and the correlation among them in Fig. 6. For the purposes of illustration we have included the histograms of the main two discriminating observables of each subanalysis in Fig. 7. Without access to the ATLAS detector simulation a direct comparison between the two approaches is not feasible. However, it can be inferred from Fig. 5 that for $0.16>\varepsilon_{2 c}$ the jet-shapes strategy shows a strong performance and is likely to add to the tagging strategy employed by ATLAS. Consequently, using event shapes it is possible to outperform the double application of a charm tagger by a single application of a doublecharm tagger. This is achieved by looking at the full radiation profile inside a fat jet, without disentangling the radiation signatures of the $c$-quark and the $\bar{c}$-quark independently.

\section{CP-ODD THDM SCALAR}

The coupling between the $C P$-odd THDM scalar $A$ and the pair $c \bar{c}$ is directly proportional to the charm quark mass $m_{c}$ and inversely proportional to the THDM vacuum ratio $\tan \beta$. As shown in [51], the decay channel $A \rightarrow c \bar{c}$ is expected to be dominant for $4.0 \mathrm{GeV} \lesssim m_{A} \lesssim 10.0 \mathrm{GeV}$ and low values of $\tan \beta$. Here we determine a 95\% C.L.

TABLE VII. Fraction of events in each one of the leptonic categories for the samples $p p \rightarrow H(\rightarrow A(\rightarrow c \bar{c}) Z)+$ jets and $p p \rightarrow Z+$ jets after the selection cuts for $m_{A}=10 \mathrm{GeV}$.

\begin{tabular}{lcc}
\hline \hline & $p p \rightarrow H+$ jets & \\
& with $H \rightarrow A(\rightarrow c \bar{c}) Z$ & \\
Fraction of events & $m_{A}=10 \mathrm{GeV}$ & $p p \rightarrow Z+$ jets \\
\hline$f^{(0)}$ (zero leptons) & $92.1 \%$ & $84.0 \%$ \\
$f^{(1)}$ (one lepton) & $7.6 \%$ & $15.15 \%$ \\
$f^{(2)}$ (two leptons) & $0.3 \%$ & $0.85 \%$ \\
\hline \hline
\end{tabular}

upper bound for the branching ratio $\mathcal{B} r(H \rightarrow A(\rightarrow c \bar{c}) Z)$ in this mass range. Our signal is the process $p p \rightarrow$ $H(\rightarrow A(\rightarrow c \bar{c}) Z)+$ jets and our background is given by $p p \rightarrow Z+$ jets. For $m_{A}=4.0 \mathrm{GeV}$ the combination of observables that gives the best performance is presented in Table IV; from here the ROCs corresponding to the different leptonic categories are determined; see Fig. 8. The optimal selection efficiency point is

$$
\varepsilon_{c \bar{c}, m_{A}=4 \mathrm{GeV}}=0.81 \quad \varepsilon_{\mathrm{QCD}, \text { jets }}=0.01
$$

resulting from the efficiencies

$$
\begin{array}{lll}
\varepsilon_{S .}^{(0)}=0.83, & \varepsilon_{S .}^{(1)}=0.69, & \varepsilon_{S .}^{(2)}=0.39 \\
\varepsilon_{B .}^{(0)}=0.01, & \varepsilon_{B .}^{(1)}=0.01, & \varepsilon_{B .}^{(2)}=0.01
\end{array}
$$

combined with the leptonic fractions presented in Table $\mathrm{V}$ as given in Eq. (1). Thus, we get the following 95\% C.L. upper limit for the branching ratio $\mathcal{B} r(H \rightarrow A(\rightarrow c \bar{c}) Z)<$ $0.01 \%$, leading to the cross section for the signal process $\sigma_{p p \rightarrow H(\rightarrow A(\rightarrow c \bar{c}) Z)+\text { jets }}=0.02 \mathrm{fb}$. For comparison, using track-based substructure observables and considering $m_{A}=$ $4.0 \mathrm{GeV}$, the $95 \%$ C.L. bound $\operatorname{Br}(H \rightarrow A(\rightarrow c \bar{c}) Z) \leq$ $2.1 \%$ has been previously determined in [20].

For $m_{A}=10 \mathrm{GeV}$ the observables per leptonic category that yield the best selection efficiency curves, shown in Fig. 9, are presented in Table VI. Our optimal result corresponds to

$$
\varepsilon_{c \bar{c}, m_{A}=10 \mathrm{GeV}}=0.38 \quad \varepsilon_{\mathrm{QCD}, \mathrm{jets}}=0.0004
$$

calculated from the individual efficiencies per leptonic category

$$
\begin{aligned}
& \varepsilon_{S .}^{(0)}=0.39, \quad \varepsilon_{S .}^{(1)}=0.29, \quad \varepsilon_{S .}^{(2)}=0.19 \\
& \varepsilon_{B .}^{(0)}=0.9 \times 10^{-4}, \quad \varepsilon_{B .}^{(1)}=14.9 \times 10^{-4}, \\
& \varepsilon_{B .}^{(2)}=177.0 \times 10^{-4},
\end{aligned}
$$




\section{Correlation Matrix (signal)}

pp $->\mathrm{H}(->\mathrm{A}(->\mathrm{cc}) \mathrm{Z})$ jets (Signal $\mathrm{m} \_\mathrm{A}=4.0 \mathrm{GeV}$, non leptonic) pp $->Z+$ jets (Background, non leptonic)

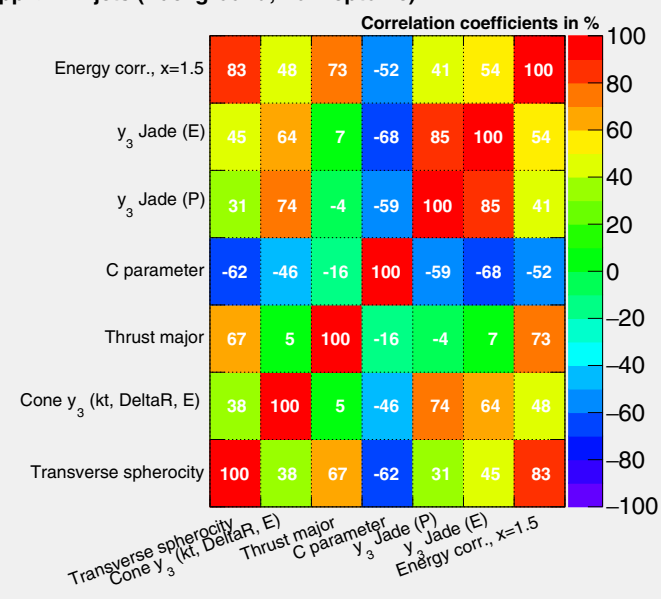

\section{Correlation Matrix (signal)}

pp $->\mathrm{H}\left(->\mathrm{A}(->\mathrm{cc}) \mathrm{Z}\right.$ ) jets (Signal $\mathrm{m}_{-} \mathrm{A}=4.0 \mathrm{GeV}$, single leptonic) pp $->Z+$ jets (Background, single leptonic)

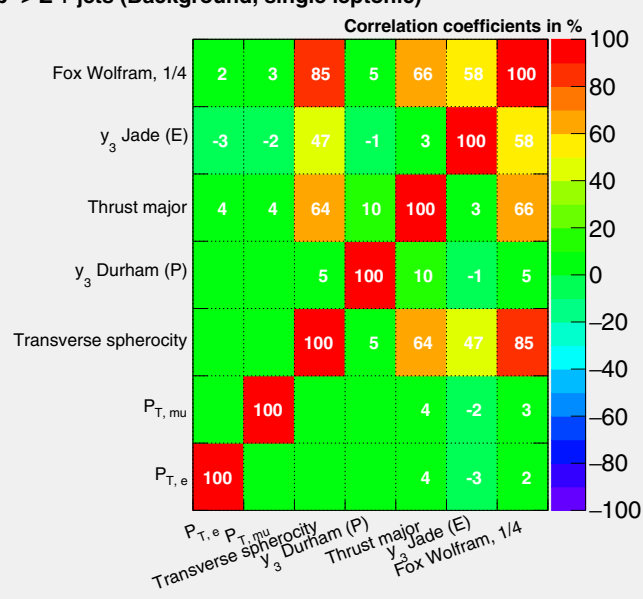

\section{Correlation Matrix (signal)}

pp $->\mathrm{H}\left(->\mathrm{A}(->\mathrm{cc}) \mathrm{Z}\right.$ ) jets (Signal $\mathrm{m} \_\mathrm{A}=4.0 \mathrm{GeV}$, double leptonic) pp $->Z+$ jets (Background, double leptonic)

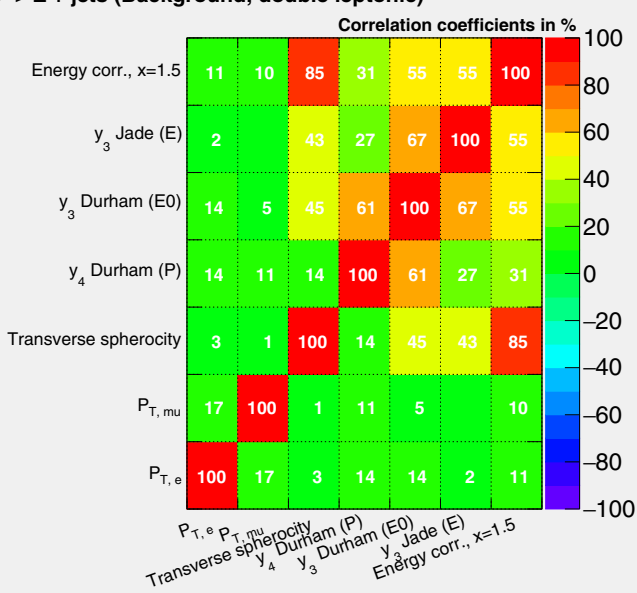

\section{Correlation Matrix (background)}

pp $->H\left(->A(->c c) Z\right.$ ) jets (Signal $m \_A=4.0 \mathrm{GeV}$, non leptonic) pp $->Z+$ jets (Background, non leptonic)

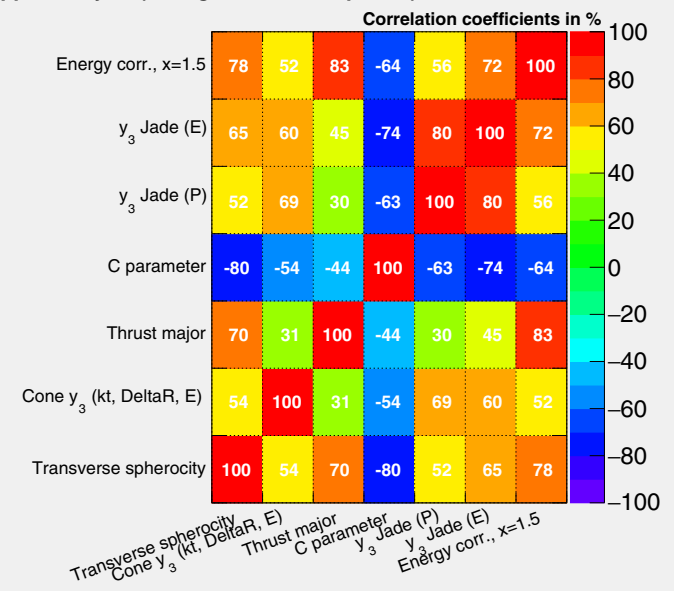

\section{Correlation Matrix (background)}

pp $->\mathrm{H}(->\mathrm{A}(->\mathrm{cc}) \mathrm{Z})$ jets (Signal $\mathrm{m} \_\mathrm{A}=4.0 \mathrm{GeV}$, single leptonic) pp $->Z+$ jets (Background, single leptonic)

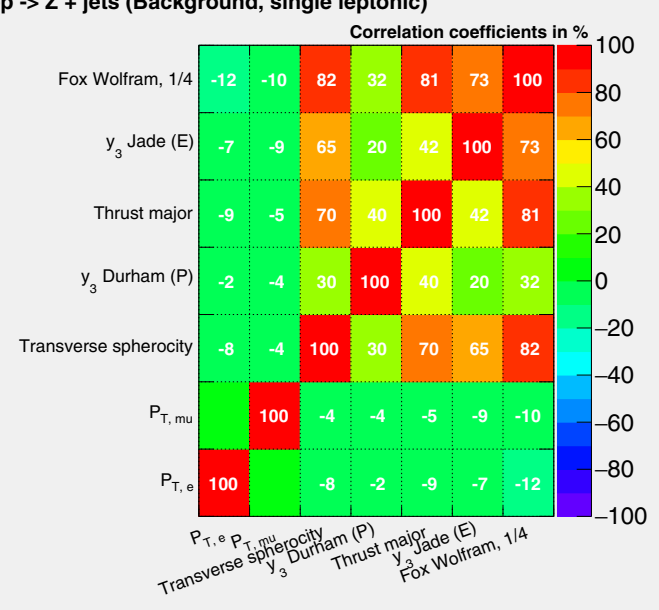

\section{Correlation Matrix (background)}

pp $->\mathrm{H}\left(->\mathrm{A}(->\mathrm{cc}) \mathrm{Z}\right.$ ) jets (Signal $\mathrm{m} \_\mathrm{A}=4.0 \mathrm{GeV}$, double leptonic) pp $->Z+$ jets (Background, double leptonic)

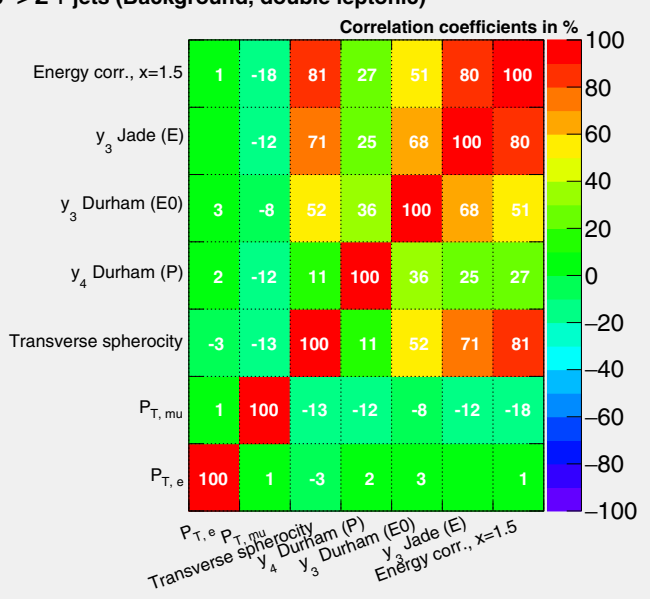

FIG. 10. Correlation coefficients for the observables used in the analysis of the $C P$-odd THDM scalar $A$, for $m_{A}=4 \mathrm{GeV}$. 


\section{Correlation Matrix (signal)}

pp $->\mathrm{H}(->\mathrm{A}(->\mathrm{cc}) \mathrm{Z})$ jets (Signal $\mathrm{m} \_\mathrm{A}=10.0 \mathrm{GeV}$, non leptonic) pp $->Z+$ jets (Background, non leptonic)

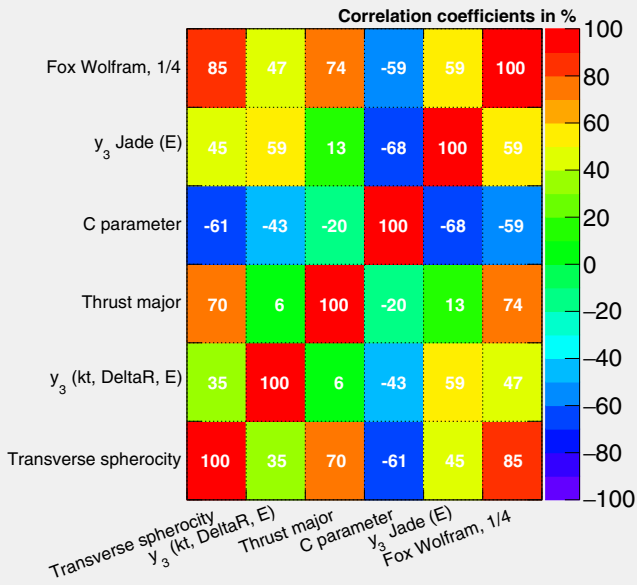

\section{Correlation Matrix (signal)}

pp $->\mathrm{H}\left(->\mathrm{A}(->\mathrm{cc}) \mathrm{Z}\right.$ ) jets (Signal $\mathrm{m} \_\mathrm{A}=10.0 \mathrm{GeV}$, single leptonic) pp $\rightarrow Z+$ jets (Background, single leptonic)

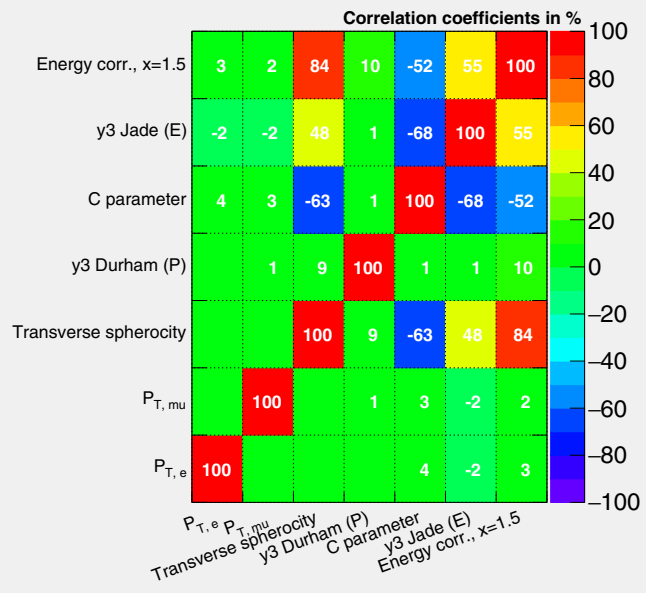

\section{Correlation Matrix (signal)}

pp $\rightarrow \mathrm{H}\left(->\mathrm{A}(->\mathrm{cc}) \mathrm{Z}\right.$ ) jets (Signal $\mathrm{m} \_\mathrm{A}=\mathbf{1 0 . 0} \mathrm{GeV}$, double leptonic) pp $->Z+$ jets (Background, double leptonic)

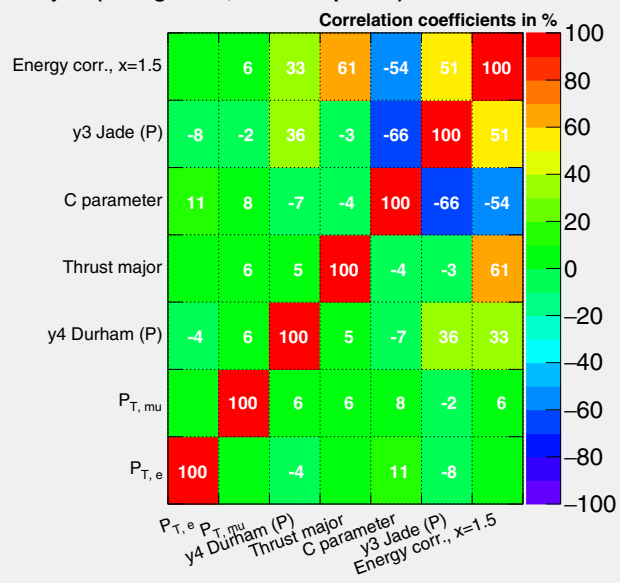

\section{Correlation Matrix (background)}

pp $->H\left(->A(->c c) Z\right.$ ) jets (Signal $m \_A=10.0 \mathrm{GeV}$, non leptonic) pp $->Z+$ jets (Background, non leptonic)

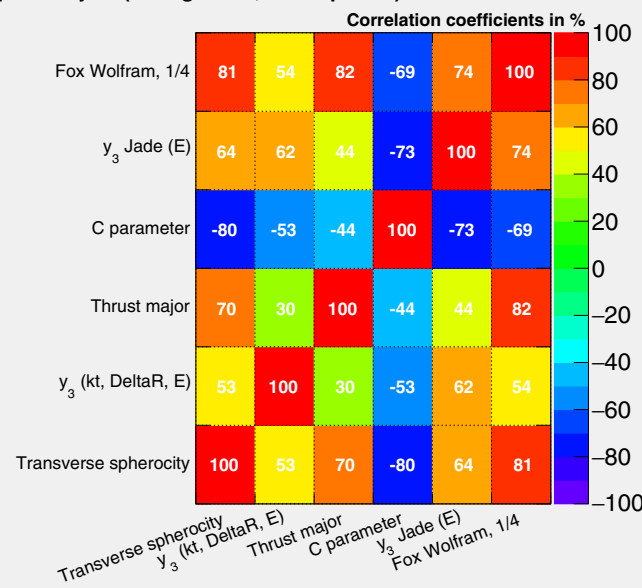

\section{Correlation Matrix (background)}

pp $->\mathrm{H}\left(->A(->c c)\right.$ Z) jets (Signal $m \_A=10.0 \mathrm{GeV}$, single leptonic) pp $->Z+$ jets (Background, single leptonic)

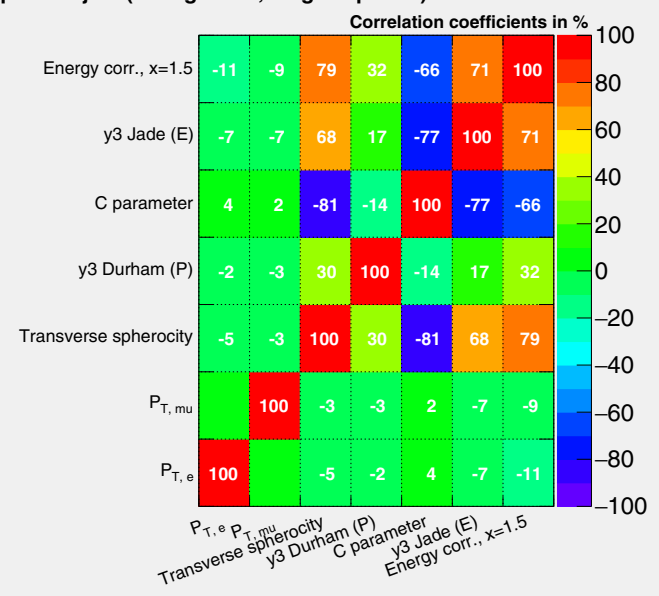

\section{Correlation Matrix (background)}

pp $->H\left(->A(->c c) Z\right.$ ) jets (Signal $m \_A=10.0 \mathrm{GeV}$, double leptonic) pp $->Z+$ jets (Background, double leptonic)

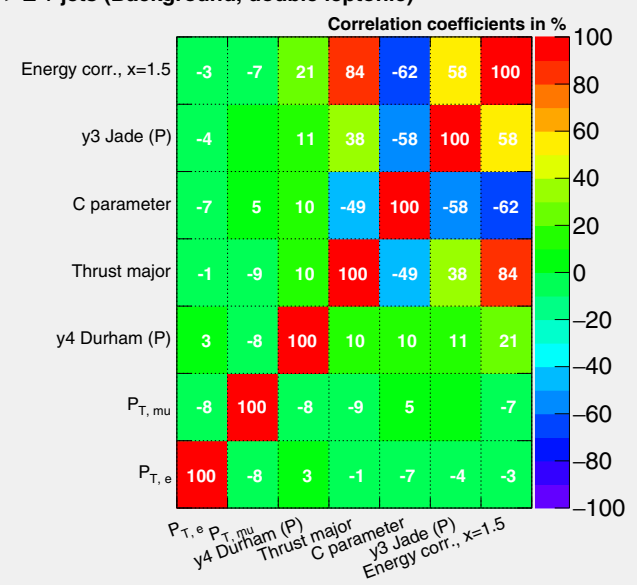

FIG. 11. Correlation coefficients for the observables used in the analysis of the $C P$-odd THDM scalar $A$, for $m_{A}=10 \mathrm{GeV}$. 
Non Leptonic Analysis $(\mathrm{mA}=4.0 \mathrm{GeV})$
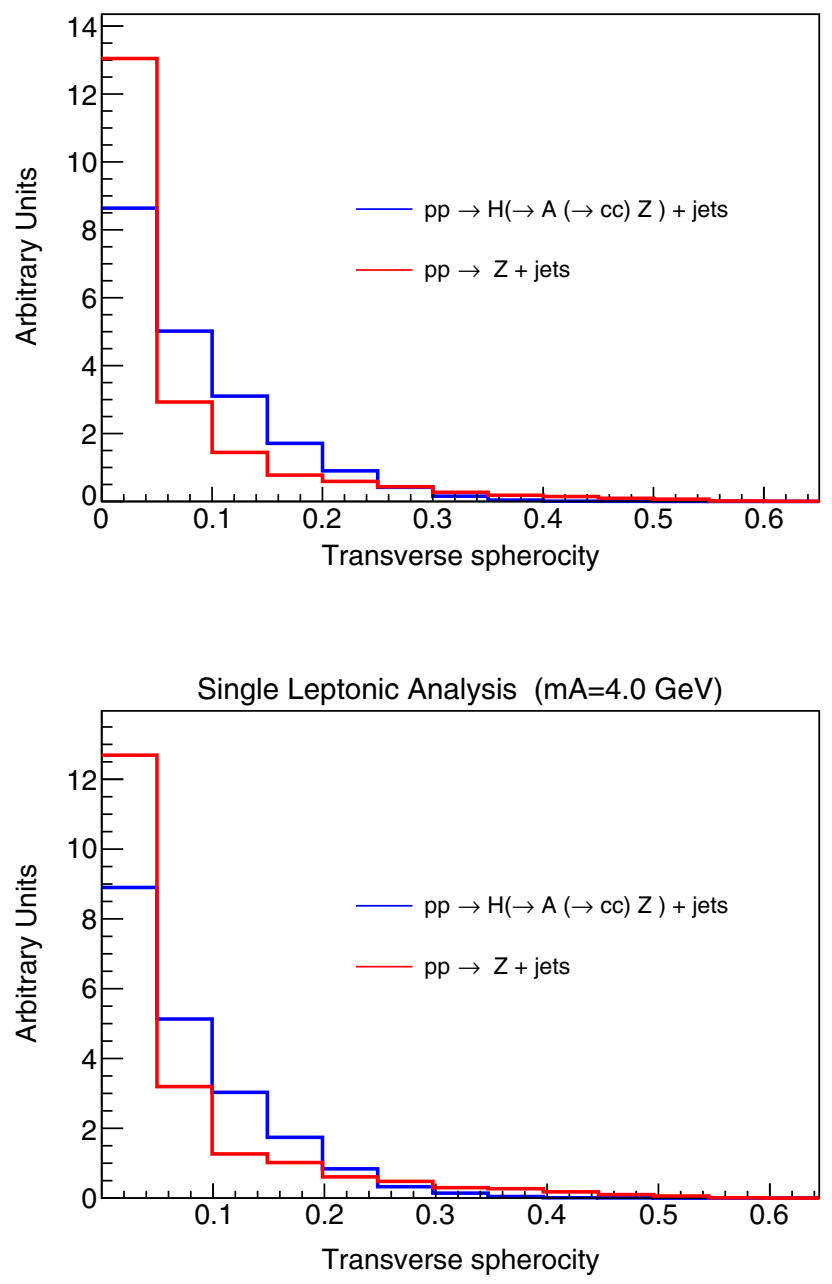

Double Leptonic Analysis $(\mathrm{mA}=4.0 \mathrm{GeV})$

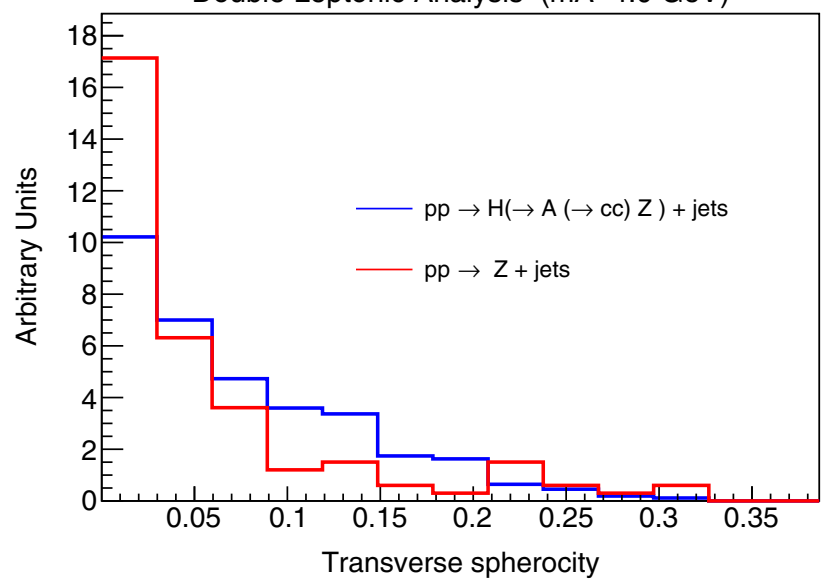

Non Leptonic Analysis $(\mathrm{mA}=4.0 \mathrm{GeV})$
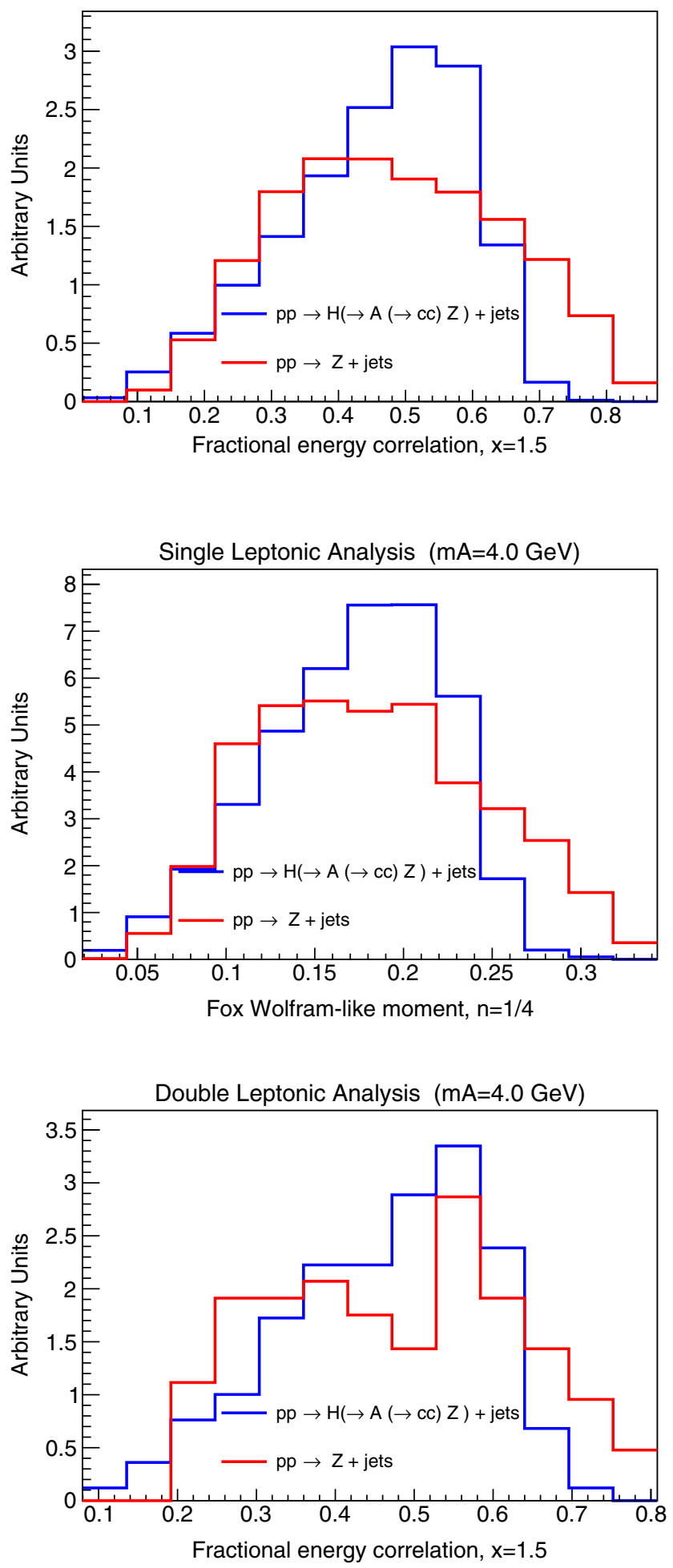

FIG. 12. Histograms for the main discrimination event shapes when selecting $p p \rightarrow H(\rightarrow A(\rightarrow c \bar{c}) Z)+$ jets against $p p \rightarrow Z+$ jets for $m_{A}=4 \mathrm{GeV}$. 

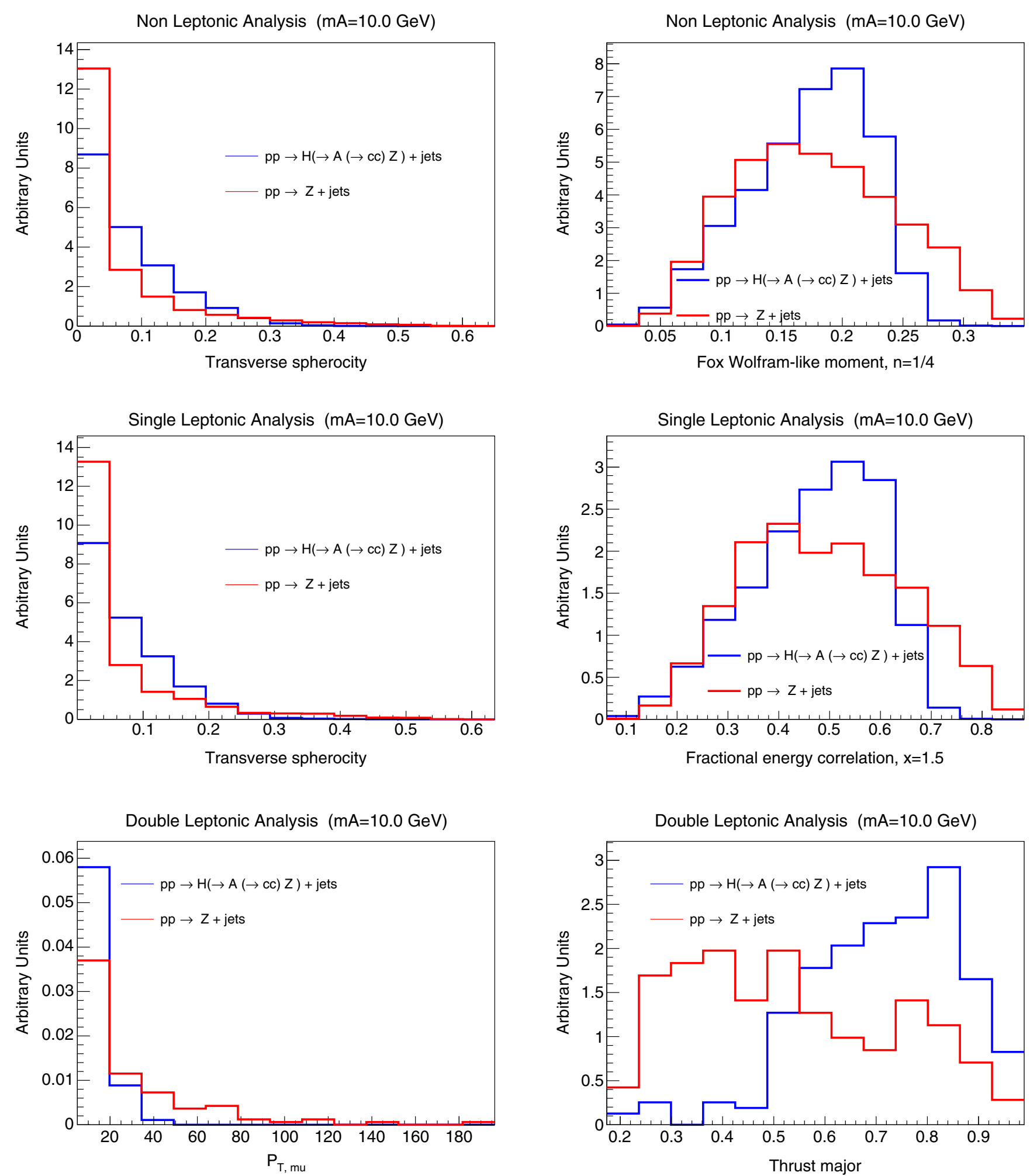

FIG. 13. Histograms for the main discrimination event shapes when selecting $p p \rightarrow H(\rightarrow A(\rightarrow c \bar{c}) Z)+$ jets against $p p \rightarrow Z+$ jets for $m_{A}=10 \mathrm{GeV}$. 
and the partial fractions of leptonic events presented in Table VII. The $95 \%$ C.L. on the branching ratio is $\mathcal{B} r(H \rightarrow A(\rightarrow c \bar{c}) Z) \leq 0.003 \%$, leading to the signal cross section $\sigma_{p p \rightarrow H(\rightarrow A(\rightarrow c \bar{c}) Z)+\text { jets }}=0.01 \mathrm{fb}$. The correlation matrices for the analyses of this section and the histograms for the main discriminating observables are shown in Figs. 10 and 11 and Figs. 12 and 13, respectively.

\section{CONCLUSIONS AND OUTLOOK}

We have studied the efficiency of event shapes for tagging jets resulting from $c \bar{c}$ pairs originated in the decay $H \rightarrow c \bar{c}$. The results obtained can be considered as an optimal limit for the performance of our selection strategy as we have not included detector effects. We have optimized our analysis depending on the main backgrounds in the selected processes and have taken into account the following possibilities: $p p \rightarrow q \bar{q} Z$ for $q=\{u, d, s, c\}, p p \rightarrow g g Z$, and $p p \rightarrow$ $H(\rightarrow b \bar{b}) Z$. Our signal channel is $p p \rightarrow H(\rightarrow c \bar{c}) Z$ and we select highly boosted Higgs bosons. Using jet-shape observables as input to a BDT, we find a good performance to separate the $c \bar{c}$ signal from $b \bar{b}$ and light-flavor fat jets.

Thus, with this approach we can project an upper limit on $\operatorname{Br}(H \rightarrow c \bar{c}) \leq 6.45 \%$ with SM production rates for $\int \mathcal{L} d t=3000.0 \mathrm{fb}^{-1}$ and $\sqrt{s}=13.0 \mathrm{TeV}$.

Following an analogous strategy we have studied the $C P$ odd THDM scalar $A$ decaying into pairs $c \bar{c}$. In particular, we have determined $\mathcal{B r}(H \rightarrow A(\rightarrow c \bar{c}) Z) \leq 0.01 \%$ by considering masses for $A$ inside the range $4.0 \mathrm{GeV} \lesssim m_{A} \lesssim$ $10.0 \mathrm{GeV}$ where the channel $A \rightarrow c \bar{c}$ is particularly dominant for low values of $\tan \beta$. The LHCb experiment has a good sensitivity towards the identification of $b$ and $c$ jets $[52,53]$ and has performed direct searches for the decay channels $H \rightarrow c \bar{c}$ and $H \rightarrow b \bar{b}$ [54]. Moreover, the possibility of detecting low mass particles similar to the scalar and pseudoscalars mentioned in this paper has already been discussed in [55]. Hence extending the present study to the realm of the $\mathrm{LHCb}$ experiment is a feasible target to be explored in more detail in a forthcoming project.

\section{ACKNOWLEDGMENTS}

M. S. is supported in part by the European Commission through the HiggsTools Initial Training Network Grant No. PITN-GA-2012-316704. We thank Silvan Kuttimalai, Nathan Hartland, and Andreas Papaefstathiou for useful discussions on SHERPA, multivariate analyses, and collider phenomenology, respectively. We also acknowledge Ian Moult and Wouter Waalewijn for pointing out analytical studies on correlations between jet-substructure observables and to Eric Laenen for interesting discussions on the topic as well.

\section{APPENDIX: EVENT SHAPES}

This section summarizes most of the observables considered during our analysis. For a more extensive discussion see $[35,56,57]$ and the references cited therein.

We begin by introducing the definition of thrust $[58,59]$,

$$
T=1-\max \left(\frac{\sum_{i}\left|\vec{p}_{i} \cdot \vec{n}_{T}\right|}{\sum_{i}\left|\vec{p}_{i}\right|}\right),
$$

where $\vec{n}_{T}$ is the direction that maximizes the numerator. To avoid confusion, in the subsequent discussion the symbol " $\perp$ " is used to denote the transverse contribution of different kinematical variables. Then, the thrust major is determined according to [60]

$$
T_{M}=\max _{\vec{n} \cdot \vec{n}_{T}=0}\left(\frac{\sum_{i}\left|\vec{p}_{i} \cdot \vec{n}\right|}{\sum_{i}\left|\vec{p}_{i}\right|}\right),
$$

where it should be understood that $\vec{n}$ is perpendicular to $\vec{n}_{T}$.

We use the thrust of $e^{-\eta}$ momenta [57] calculated according to Eq. (A1) but with the three-momenta of each one of the subjets in the event modified according to

$$
\vec{p}_{i} \rightarrow \vec{p}_{i} e^{-\left|\eta_{i}\right|}
$$

We include the Fox Wolfram moment inspired observable [61]

$$
H_{n}=\sum_{i, j} \frac{\left|\vec{p}_{i}\right|\left|\vec{p}_{j}\right|}{E_{\mathrm{Tot}}^{2}} \sin ^{2 n} \theta_{i j},
$$

with $E_{\mathrm{Tot}}$ being the total energy of the jet constituents; thus $E_{\mathrm{Tot}}=\sum_{i} E_{i}$. The sum in the numerator of Eq. (A4) considers only pairs of particles within the same hemisphere, i.e., those particles satisfying $\vec{p}_{i} \cdot \vec{p}_{j}>0$, and $n$ is a rational number. In what follows we refer to $H_{n}$ as the Fox Wolfram-like $n$ moment, and we consider the value $n=1 / 4$.

The transverse spherocity [36] is given by

$$
S_{p h}=\min \left(\frac{2}{\pi}\right)^{2} \frac{\left(\sum_{i}\left|\vec{p}_{\perp} \times \hat{n}^{\prime \prime}\right|\right)^{2}}{\left(\sum_{i}\left|\vec{p}_{\perp, i}\right|\right)^{2}}
$$

where $\hat{n}^{\prime \prime}$ is the direction that minimizes the sum in the numerator.

The 3 -jet resolution $y_{3}$ defines the lower bound for the jet recombination parameter $y_{i j}$ in order to have a 3 -jet event. Before presenting the determination algorithm for $y_{3}$, according to different schemes, let us first introduce the possible definitions for the parameter $y_{i j}$.

$$
y_{i j}= \begin{cases}2 \min \left(E_{i}^{2}, E_{j}^{2}\right)\left(1-\cos \theta_{i j}\right) / E_{\mathrm{vis}}^{2} & \text { Durham } \\ \frac{8 E_{i} E_{j}\left(1-\cos \theta_{i j}\right)}{9\left(E_{i}+E_{j}\right)^{2}} & \text { Geneva } \\ \left(p_{i}+p_{j}\right)^{2} / E_{\mathrm{vis}}^{2} & \text { Jade, }\end{cases}
$$


with $E_{\text {vis }}$ being the sum of the energies for the different final state subjets before the recombinations.

In addition, the recombination schemes between the $i$ th and $j$ th subjets are

Schemes $=\left\{\begin{array}{l}P: \vec{p}=\vec{p}_{i}+\vec{p}_{j}, E_{p}=|\vec{p}| \\ E: p=p_{i}+p_{j} \\ E 0: \vec{p}=\frac{E_{i}+E_{j}}{\left|\vec{p}_{i}+\vec{p}_{j}\right|}\left(\vec{p}_{i}+\vec{p}_{j}\right), E_{p}=E_{p, i}+E_{p, j} .\end{array}\right.$

Then for example, in order to calculate the resolution $y_{3}$ Durham (P-scheme) [62,63], we start by assigning an arbitrary high value to $y_{3}$. Next, we calculate the parameter $y_{i j}$ between all the subjets inside a given fat jet using the Durham recombination rule shown before. We then determine the pair of elements whose $y_{i j}$ is minimum, $y_{i j}^{\min }$, and recombine them applying the P-scheme presented above. Finally, if $y_{3}<y_{i j}^{\min }$, we do the substitution $y_{3}=y_{i j}^{\min }$ and repeat the entire process, starting with the recalculation of the values $y_{i j}$ over the set of subjets determined in the last iteration. The algorithm stops when the total number of subjets left after all the recombinations is equal to 3 . The value of $y_{3}$ obtained in the final iteration is the number we are aiming for. The determination of the resolution $y_{3}$ Jade (E-scheme) and the resolution $y_{3}$ Jade (E0-scheme) proceeds in an analogous way; however the Durham parameter $y_{i j}$ should be substituted by the Jade distance parameter; and the $\mathrm{P}$ recombination scheme should be replaced by the E-scheme (E0-scheme).

The directly global $y_{3}$ [36] is constructed using the $k_{t}$-jet algorithm. To begin with, for all $n$ final state particles we define the beam-distance measure

$$
d_{k, B}=p_{\perp k}^{2},
$$

and for constituent pairs we calculate

$$
d_{k l}=\min \left\{p_{\perp k}^{2}, p_{\perp l}^{2}\right\} \frac{\left(y_{k}-y_{l}\right)^{2}+\left(\phi_{k}-\phi_{l}\right)^{2}}{R^{2}},
$$

in terms of their corresponding pseudorapidity $y$ and azimuthal angle $\phi$.

In our analysis we use $R=0.7$. Let $d^{(n)}=\min \left\{d_{k B}, d_{k l}\right\}$, where the entire set of distances calculated at a given stage is considered. If $d^{(n)}$ is one of the values $d_{i j}$, then the pseudojets $i$ and $j$ are recombined using the E-scheme defined above. If $d^{(n)}$ is one of the individual coefficients $d_{k B}$, then the pseudojet is removed and included in the beam. These steps are repeated until only three pseudojets are left. At this stage we determine

$$
y_{23}=\frac{1}{P_{\perp}^{2}} \max _{n \geq 3}\left\{d^{(n)}\right\},
$$

with

$$
P_{\perp}=p_{\perp, 1}+p_{\perp, 2},
$$

$p_{\perp, 1}$ and $p_{\perp, 2}$ being the transverse momenta of the jets obtained by continuing reclustering the event up to two pseudojets.

The observable $\tau_{x}$ can be modified to give the fractional energy correlation [57]

$$
\begin{aligned}
F C_{x}= & \sum_{i \neq j} \frac{E_{i} E_{j}\left|\sin \theta_{i j}\right|\left(1-\left|\cos \theta_{i j}\right|\right)^{1-x}}{\left(\sum_{i} E_{i}\right)^{2}} \\
& \times \Theta\left[\left(\vec{p}_{i} \cdot \vec{n}_{T}\right)\left(\vec{p}_{j} \cdot \vec{n}_{T}\right)\right] .
\end{aligned}
$$

Here $x$ is a continuous parameter. During the analysis we use $x=1.5$ that makes the observable particularly sensitive to collinear emissions for fixed transverse momentum.

To define the transverse sphericity let us first introduce the transverse momentum tensor,

$$
M_{x y}=\sum_{i}\left(\begin{array}{cc}
p_{x, i}^{2} & p_{x, i} p_{y, i} \\
p_{x, i} p_{y, i} & p_{y, i}^{2}
\end{array}\right) .
$$

Then the transverse sphericity can be determined in terms of the eigenvalues $\lambda_{1}$ and $\lambda_{2}$ of $M_{x y}\left(\right.$ for $\lambda_{1} \geq \lambda_{2}$ ) as [64]

$$
S_{\perp, g}^{\text {pheri }} \equiv \frac{2 \lambda_{2}}{\lambda_{1}+\lambda_{2}}
$$

for circular events in the transverse plane we have $S_{\perp, g}^{\text {pheri }} \rightarrow 1$, whereas for pencil-like events $S_{\perp, g}^{\text {pheri }} \rightarrow 0$.

To describe the cone jet mass let us start by introducing some definitions. The components of the highest $p_{T}$ fat jet selected in our studies are first reclustered using the $k_{t}$ algorithm. Then, the region $\mathcal{C}$ results from the union of the cones around the two new highest transverse momentum subjets (with coordinates $\eta_{J, j}, \phi_{J, j}$, for $j=1,2$ ) according to

$$
\sqrt{\left(\eta_{i}-\eta_{J, j}\right)^{2}+\left(\phi_{i}-\phi_{J, j}\right)^{2}} \leq R
$$

where the subindex $i$ runs over the rest of the newly generated subjets. During our implementation we considered $R=1$. The central transverse thrust axis $\vec{n}_{T, C}$ is then defined as the vector that maximizes

$$
\frac{\sum_{i \in \mathcal{C}}\left|\vec{p}_{\perp i} \cdot \vec{n}_{T, C}\right|}{Q_{\perp, \mathcal{C}}},
$$

where 


$$
Q_{\perp, \mathcal{C}}=\sum_{i \in \mathcal{C}}\left|\vec{p}_{\perp i}\right|
$$

The vector $\vec{n}_{T, C}$ allows us to divide the region $\mathcal{C}$ into the subregions $\mathcal{C}_{U}$ and $\mathcal{C}_{D}$, defined in terms of the conditions $0<\vec{p}_{\perp} \cdot \vec{n}_{T, C}$ and $\vec{p}_{\perp} \cdot \vec{n}_{T, C}<0$, respectively. The partial masses in each one of these regions are

$$
\rho_{U, \mathcal{C}}=\frac{\left(\sum_{i \in \mathcal{C}_{U}} p_{i}\right)^{2}}{Q_{\perp, \mathcal{C}}^{2}}, \quad \rho_{D, \mathcal{C}}=\frac{\left(\sum_{i \in \mathcal{C}_{D}} p_{i}\right)^{2}}{Q_{\perp, \mathcal{C}}^{2}} .
$$

Then, the cone total jet mass is

$$
\rho_{S, \mathcal{C}}=\rho_{U, \mathcal{C}}+\rho_{D, \mathcal{C}}
$$

and the heavy jet mass is defined as

$$
\rho_{H, \mathcal{C}}=\max \left\{\rho_{U, \mathcal{C}}, \rho_{D, \mathcal{C}}\right\}
$$

Finally, we consider the following $C$ parameterlike observable [65-67],

$$
C=3-\frac{3}{E_{\mathrm{Tot}}^{2}} \sum_{i<j} \frac{\left(p_{i} \cdot p_{j}\right)^{2}}{E_{i} E_{j}}
$$

with $E_{\mathrm{Tot}}=\sum_{i} E_{i}$.
[1] G. Aad et al. (ATLAS Collaboration), Phys. Lett. B 716, 1 (2012).

[2] S. Chatrchyan et al. (CMS Collaboration), Phys. Lett. B 716, 30 (2012).

[3] V. Khachatryan et al. (CMS Collaboration), Eur. Phys. J. C 75, 212 (2015).

[4] G. Aad et al. (ATLAS Collaboration), Eur. Phys. J. C 76, 6 (2016).

[5] G. Aad et al. (ATLAS, CMS Collaborations), J. High Energy Phys. 08 (2016) 045.

[6] D. Ghosh, R. S. Gupta, and G. Perez, Phys. Lett. B 755, 504 (2016).

[7] G. Perez, Y. Soreq, E. Stamou, and K. Tobioka, Phys. Rev. D 92, 033016 (2015).

[8] M. Aaboud et al. (ATLAS Collaboration), arXiv: 1708.03299 [J. High Energy Phys. (to be published)].

[9] A.M. Sirunyan et al. (CMS Collaboration), arXiv: 1709.07497 [Phys. Lett. B (to be published)].

[10] A. M. Sirunyan et al. (CMS Collaboration), arXiv: 1708.00373 [Phys. Lett. B (to be published)].

[11] G. Aad et al. (ATLAS Collaboration), Phys. Lett. B 738, 68 (2014).

[12] V. Khachatryan et al. (CMS Collaboration), Phys. Lett. B 744, 184 (2015).

[13] CMS Collaboration, in Proceedings, 2013 Community Summer Study on the Future of U.S. Particle Physics, Snowmass on the Mississippi (CSS2013), Minneapolis, 2013 (2013) [arXiv:1307.7135].

[14] W. Altmannshofer, J. Brod, and M. Schmaltz, J. High Energy Phys. 05 (2015) 125.

[15] D. d'Enterria, in Proceedings, 17th Lomonosov Conference on Elementary Particle Physics, Moscow, Russia, 2015 (2017), pp. 182-191.

[16] G. Aad et al. (ATLAS Collaboration), Phys. Rev. Lett. 114, 121801 (2015).

[17] G. T. Bodwin, F. Petriello, S. Stoynev, and M. Velasco, Phys. Rev. D 88, 053003 (2013).
[18] M. Knig and M. Neubert, J. High Energy Phys. 08 (2015) 012.

[19] G. Perez, Y. Soreq, E. Stamou, and K. Tobioka, Phys. Rev. D 93, 013001 (2016).

[20] A. S. Chisholm, S. Kuttimalai, K. Nikolopoulos, and M. Spannowsky, Eur. Phys. J. C 76, 501 (2016).

[21] C. Delaunay, T. Golling, G. Perez, and Y. Soreq, Phys. Rev. D 89, 033014 (2014).

[22] I. Brivio, F. Goertz, and G. Isidori, Phys. Rev. Lett. 115, 211801 (2015).

[23] L. M. Carpenter, T. Han, K. Hendricks, Z. Qian, and N. Zhou, Phys. Rev. D 95, 053003 (2017).

[24] F. Bishara, U. Haisch, P. F. Monni, and E. Re, Phys. Rev. Lett. 118, 121801 (2017).

[25] G. F. Giudice and O. Lebedev, Phys. Lett. B 665, 79 (2008).

[26] F. J. Botella, G. C. Branco, M. N. Rebelo, and J. I. Silva-Marcos, Phys. Rev. D 94, 115031 (2016).

[27] R. Harnik, J. Kopp, and J. Zupan, J. High Energy Phys. 03 (2013) 026.

[28] M. Bauer, M. Carena, and K. Gemmler, Phys. Rev. D 94, 115030 (2016).

[29] W. Altmannshofer, J. Eby, S. Gori, M. Lotito, M. Martone, and D. Tuckler, Phys. Rev. D 94, 115032 (2016).

[30] F. Bishara, J. Brod, P. Uttayarat, and J. Zupan, J. High Energy Phys. 01 (2016) 010.

[31] CERN Report No. ATL-PHYS-PUB-2015-001, 2015.

[32] F. Abe et al. (CDF Collaboration), Phys. Rev. Lett. 74, 2626 (1995).

[33] S. Abachi et al. (D0 Collaboration), Phys. Rev. Lett. 74, 2422 (1995).

[34] G. Marchesini and B. R. Webber, Nucl. Phys. B238, 1 (1984).

[35] A. Banfi, G. P. Salam, and G. Zanderighi, J. High Energy Phys. 08 (2004) 062.

[36] A. Banfi, G. P. Salam, and G. Zanderighi, J. High Energy Phys. 06 (2010) 038. 
[37] C. Englert, M. Spannowsky, and M. Takeuchi, J. High Energy Phys. 06 (2012) 108.

[38] C. Bernaciak, M. S. A. Buschmann, A. Butter, and T. Plehn, Phys. Rev. D 87, 073014 (2013).

[39] A. J. Larkoski, J. Thaler, and W. J. Waalewijn, J. High Energy Phys. 11 (2014) 129.

[40] A. J. Larkoski, I. Moult, and D. Neill, J. High Energy Phys. 09 (2014) 046.

[41] M. Procura, W. J. Waalewijn, and L. Zeune, J. High Energy Phys. 02 (2015) 117.

[42] I. Moult, B. Nachman, and D. Neill, arXiv:1710.06859.

[43] C. Shimmin, P. Sadowski, P. Baldi, E. Weik, D. Whiteson, E. Goul, and A. Sgaard, Phys. Rev. D 96, 074034 (2017).

[44] J. A. Aguilar-Saavedra, J. H. Collins, and R. K. Mishra, J. High Energy Phys. 11 (2017) 163.

[45] T. Gleisberg, S. Hoeche, F. Krauss, M. Schonherr, S. Schumann, F. Siegert, and J. Winter, J. High Energy Phys. 02 (2009) 007.

[46] M. Cacciari, G. P. Salam, and G. Soyez, Eur. Phys. J. C 72, 1896 (2012).

[47] A. Buckley, J. Butterworth, L. Lonnblad, D. Grellscheid, H. Hoeth, J. Monk, H. Schulz, and F. Siegert, Comput. Phys. Commun. 184, 2803 (2013).

[48] J. Barnard, E. N. Dawe, M. J. Dolan, and N. Rajcic, Phys. Rev. D 95, 014018 (2017).

[49] F. Krinner, A. Lenz, and T. Rauh, Nucl. Phys. B876, 31 (2013).

[50] A. Hocker et al., Proc. Sci., ACAT2007 (2007) 040 [arXiv: physics/0703039].

[51] R. Dermisek and J. F. Gunion, Phys. Rev. D 81, 075003 (2010).
[52] R. Aaij et al. (The LHCb collaboration), J. Instrum. 10, P06013 (2015).

[53] P. Ilten, N. L. Rodd, J. Thaler, and M. Williams, Phys. Rev. D 96, 054019 (2017).

[54] The LHCb Collaboration, Reports No. LHCb-CONF-2016006, No. CERN-LHCb-CONF-2016-006 2016.

[55] R. Aaij et al. (LHCb Collaboration), Eur. Phys. J. C 77, 812 (2017).

[56] A. Banfi, G. P. Salam, and G. Zanderighi, Phys. Lett. B 584, 298 (2004).

[57] A. Banfi, G. P. Salam, and G. Zanderighi, J. High Energy Phys. 03 (2005) 073.

[58] S. Brandt, C. Peyrou, R. Sosnowski, and A. Wroblewski, Phys. Lett. 12, 57 (1964).

[59] E. Farhi, Phys. Rev. Lett. 39, 1587 (1977).

[60] D. P. Barber et al. (MARK-J, AACHEN-DESY-MITNIKHEF-BEIJING Collaborations), Phys. Rep. 63, 337 (1980).

[61] G. C. Fox and S. Wolfram, Phys. Rev. Lett. 41, 1581 (1978).

[62] S. Catani, Y. L. Dokshitzer, M. Olsson, G. Turnock, and B. R. Webber, Phys. Lett. B 269, 432 (1991).

[63] A. Banfi, G. P. Salam, and G. Zanderighi, J. High Energy Phys. 01 (2002) 018.

[64] J. D. Bjorken and S. J. Brodsky, Phys. Rev. D 1, 1416 (1970).

[65] G. Parisi, Phys. Lett. 74B, 65 (1978).

[66] J. F. Donoghue, F. E. Low, and S.-Y. Pi, Phys. Rev. D 20, 2759 (1979).

[67] S. Catani and B. R. Webber, Phys. Lett. B 427, 377 (1998). 